\title{
Some New Coupled Fixed-Point Findings Depending on Another Function in Fuzzy Cone Metric Spaces with Application
}

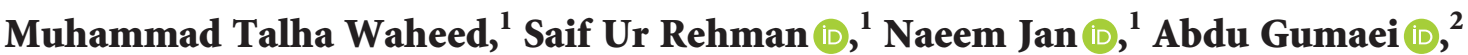 \\ and Mabrook Al-Rakhami ${ }^{3}{ }^{3}$ \\ ${ }^{1}$ Department of Mathematics, Gomal University, Dera Ismail Khan 29050, Pakistan \\ ${ }^{2}$ Computer Science Department, Faculty of Applied Sciences, Taiz University, Taiz 6803, Yemen \\ ${ }^{3}$ STC's Artificial Intelligence Chair, Department of Information Systems, King Saud University, Riyadh 11543, Saudi Arabia
}

Correspondence should be addressed to Abdu Gumaei; abdugumaei@taiz.edu.ye and

Mabrook Al-Rakhami; malrakhami@ksu.edu.sa

Received 1 May 2021; Accepted 16 June 2021; Published 8 July 2021

Academic Editor: G. Muhiuddin

Copyright ( $) 2021$ Muhammad Talha Waheed et al. This is an open access article distributed under the Creative Commons Attribution License, which permits unrestricted use, distribution, and reproduction in any medium, provided the original work is properly cited.

In this paper, we introduce the new concept of coupled fixed-point (FP) results depending on another function in fuzzy cone metric spaces (FCM-spaces) and prove some unique coupled FP theorems under the modified contractive type conditions by using "the triangular property of fuzzy cone metric." Another function is self-mapping continuous, one-one, and subsequently convergent in FCM-spaces. In support of our results, we present illustrative examples. Moreover, as an application, we ensure the existence of a common solution of the two Volterra integral equations to uplift our work.

\section{Introduction}

Fixed-point theory is one of the most interesting areas of research. In 1922, Banach [1] proved a "Banach contraction principle" stated as follows: "a single-valued contractive type mapping in a complete metric space has a unique FP." After the publication of this principle, many researchers have contributed their ideas to the problems on fixed points in the context of metric spaces for single-valued and multivalued mappings with different types of applications. Kannan [2] and Chatterjea [3] proved some fixed-point theorems, while Reich $[4,5]$ presented some remarks concerning contractive type mappings in complete metric spaces. Covitz and Nadler [6] and Daffer and Kaneko [7] proved some multivalued fixedpoint theorems, while Kaewkhao and Neammanee [8] established fixed-point theorems for multivalued Zamfirescu mapping in complete metric spaces. In 2007, Huang and Zhang [9] introduced the notion of cone metric space in which they extended and modified the concept of metric spaces. They proved the convergence properties and some fixed-point results by using the concept of the underlying cone are normal.
Meanwhile, in 2008, Rezapour and Hamlbarani [10] proved fixed-point theorems without the assumption of normality of cone. After that, many others contributed their ideas to the problems on fixed-point results in cone metric spaces. Some of their contributions to the problems on cone metric spaces for fixed points can be found in [11-14].

Initially, the concept of fuzzy set theory was given by Zadeh [15]. Recently, the fuzzy set theory has been investigated, applied, and modified in many directions, in which the one direction of this theory is fuzzy logic, which has a wide range of applications again in many directions such as in engineering fields, business, and education. In education, fuzzy logic is used for the student results evaluation, which can be directly monitored by the teacher. Some of the references related to an education system based on fuzzy logic can be found in [16-19]. The other direction of the fuzzy set is the fuzzy metric theory. The notion of FM-space was introduced by Kramosil and Michalek [20]; they used the concept of a fuzzy set on metric space and proved some basic properties of the FM-space. After that, the stronger form of the metric fuzziness was given by George and Veeramani [21]. Later on, 
Gregori and Sapena [22] proved some contractive type FP theorems in FM-spaces. Recently, in 2020, Li et al. [23] proved some strongly coupled FP theorems by using cyclic contractive type mappings in complete FM-spaces. Meanwhile Rehman et al. [24] presented the concept of rational type contraction mappings and proved some FP theorems in complete FMspaces with an application.

In 2015, Oner et al. [25] introduced the concept of fuzzy cone metric spaces (FCM-spaces) and proved some basic properties and "a single-valued Banach contraction theorem for FP with the assumption that all the sequences are Cauchy." Later on, Rehman and $\mathrm{Li}$ [26] established some generalized fuzzy cone-contractive type results for FP without the assumption that "all the sequences are Cauchy." After that, Jabeen et al. [27] proved common FP theorems for quasi-contraction by using the concept of compatible and weakly compatible for three self-mappings with an integral type application. In 2020, Chen et al. [28] introduced the concept of coupled contractive type mappings in FCM-spaces and proved some coupled FP results with application to nonlinear integral type application. Recently, in 2021, Rehman and Aydi [29] proved some rational type common FP theorems in FCM-spaces with an application.

In [30], Guo and Lakshmikantham introduced the coupled FP results for the nonlinear operator with applications. After that, some coupled FP theorems in partially ordered metric spaces were proved by Bhaskar and Lakshmikantham [31] and Lakshmikantham and Ciric [32]. In 2010, Sedghi et al. [33] proved common coupled FP theorems for commuting mappings in FM-spaces. Meanwhile Moradi [34] presented some results on "Kannan FP on complete and generalized metric spaces which depends on another function" by using the concept of subsequence convergence and continuity.

In this paper, we use the above concepts together and prove some unique coupled FP theorems depending on another function in FCM-spaces. Moreover, we present an application of the two Volterra integral equations for a common solution to support our results. This new concept will play an important role in the theory of fixed point to prove more coupled FP and strongly coupled FP results in complete FCM-spaces with the application of different types of differential equations. This paper is organized as follows: Section 2 gives preliminary concepts. In Section 3, we use the concepts of Guo and Lakshmikantham [30], Moradi [34], Chen et al. [28], and Jabeen et al. [27] all together and establish some unique coupled FP results depending on another continuous function which is one-one and subsequently convergent in FCM-spaces. In Section 4, we present an application of the two Volterra integral equations for the existence of a common solution to support our main work. In the last section (Section 5 ), we present the conclusion of our work.

\section{Preliminaries}

Definition 1. Let $G$ be any set. $A$ fuzzy set $A$ in $G$ is a function whose domain is $G$ and the range is $[0,1]$.

Definition 2 (see [35]). A binary operation $*$ : $[0,1] \times$ $[0,1] \longrightarrow[0,1]$ would be a continuous $t$-norm if $*$ fulfils the following conditions: (i) * is associative and commutative

(ii) $*$ is continuous

(iii) $1 * \alpha=\alpha, \forall \alpha \in[0,1]$

(iv) $\alpha * \beta \leq \gamma * \delta$ whenever $\alpha \leq \gamma$ and $\beta \leq \delta$, for $\alpha$, $\beta, \gamma, \delta \in[0,1]$

Definition 3 (see [9]). Let $E$ be a real Banach space, and $P$ is a subset of $E$. Then, $P$ is called a cone if

(i) $P$ is closed and nonempty and $P \neq\{0\}$

(ii) If $\alpha, \beta \in R, \alpha, \beta \geq 0$ and $g, h \in P$, then $\alpha g+\beta h \in P$

(iii) If both $g \in P$ and $-g \in P$, then $g=0$

A partial ordering on a given cone $P \subset E$ is defined by $g \preceq h \Leftrightarrow h-g \in P . g \preceq h$ stands for $g \preceq h$ and $g \neq h$, while $g \ll h$ stands for $h-g \in \operatorname{int}(P)$. In this paper, all cones have a nonempty interior.

Definition 4 (see [21]). A 3-tuple $\left(G, M_{c}, *\right)$ is said to be an FM-space if $G$ is any set, $*$ is continuous $t$-norm, and $M_{c}$ is a fuzzy set on $G^{2} \times(0, \infty)$ satisfying

(i) $M_{c}(g, h, t)>0$

(ii) $M_{c}(g, h, t)=0$ if and only if $g=h$

(iii) $M_{c}(g, h, t)=M_{c}(h, g, t)$

(iv) $M_{c}(g, k, t)+M_{c}(k, h, s) \leq M_{c}(g, h, t+s)$

(v) $M_{c}(g, h, \cdot):(0, \infty) \longrightarrow[0,1]$ is continuous, for $g, h, k \in G$ and $t, s>0$

Definition 5 (see [25]). A 3-tuple $\left(G, M_{c}, *\right)$ is said to be an FCM-space if $P$ is a cone of $E, G$ is an arbitrary set, * is continuous $t$-norm, and $M_{c}$ is a fuzzy set on $G^{2} \times \operatorname{int}(P)$ satisfying

(i) $M_{c}(g, h, t)>0$

(ii) $M_{c}(g, h, t)=0$ if and only if $g=h$

(iii) $M_{c}(g, h, t)=M_{c}(h, g, t)$

(iv) $M_{c}(g, k, t)+M_{c}(k, h, s) \leq M_{c}(g, h, t+s)$

(v) $M_{c}(g, h, \cdot): \operatorname{int}(P) \longrightarrow[0,1]$ is continuous, for $g, h, k \in G$, and $t, s \gg 0$

Definition 6 (see [25]). Let a 3-tuple $\left(G, M_{c}\right.$, * ) be an FCMspace, $h_{1} \in G$, and a sequence $\left\{h_{\ell}\right\}$ in $G$ is

(i) Converging to $h_{1}$ if $\gamma \in(0,1)$ and $t \gg 0$ and there is $\ell_{1} \in \mathbb{N}$ such that $M_{c}\left(h_{\ell}, h_{1}, t\right)>1-\gamma$, for $\ell \geq \ell_{1}$. We may write this $\lim _{\ell \longrightarrow \infty} h_{\ell}=h_{1}$ or $h_{\ell} \longrightarrow h_{1}$ as $\ell \longrightarrow \infty$;

(ii) Cauchy sequence if $\gamma \in(0,1)$ and $t \gg 0$ and there is $\ell_{1} \in \mathbb{N}$ such that $M_{c}\left(h_{\ell}, h_{j}, t\right)>1-\gamma$, for $\ell, j \geq \ell_{1}$;

(iii) $\left(G, M_{c}, *\right)$ complete if every Cauchy sequence is convergent in $G$;

(iv) Fuzzy cone contractive if $\exists \alpha \in(0,1)$, satisfying

$\frac{1}{M_{c}\left(h_{\ell}, h_{\ell+1}, t\right)}-1 \leq \alpha\left(\frac{1}{M_{c}\left(h_{\ell-1}, h_{\ell}, t\right)}-1\right)$,

for $t \gg 0, \ell \geq 1$. 
Lemma 1 (see [25]). Let $\left(G, M_{c}\right.$, *) be an FCM-space and let a sequence $\left\{h_{\ell}\right\}$ in $G$ converge to a point $h_{1} \in G$ if $M_{c}\left(h_{\ell}, h_{1}, t\right) \longrightarrow 1$ as $\ell \longrightarrow \infty$, for $t \gg 0$.

Definition 7 (see [26]). Let $\left(G, M_{c}\right.$, *) be an FCM-space. The fuzzy cone metric $M_{c}$ is triangular if

$$
\begin{array}{r}
\frac{1}{M_{c}\left(h_{1}, h_{3}, t\right)}-1 \leq\left(\frac{1}{M_{c}\left(h_{1}, h_{2}, t\right)}-1\right)+ \\
+\left(\frac{1}{M_{c}\left(h_{2}, h_{3}, t\right)}-1\right), \\
\forall h_{1}, h_{2}, h_{3} \in G, t \gg 0 .
\end{array}
$$

Definition 8 (see [25]). Let $\left(G, M_{c}, *\right)$ be an FCM-space and $\dot{A}: G \longrightarrow G$. Then $\dot{A}$ is said to be fuzzy cone contractive if there exists $\alpha \in(0,1)$ such that

$$
\begin{array}{r}
\frac{1}{M_{c}\left(\dot{A} h_{1}, \dot{A} h_{2}, t\right)}-1 \leq \alpha\left(\frac{1}{M_{c}\left(h_{1}, h_{2}, t\right)}-1\right), \\
\forall h_{1}, h_{2} \in G, t \gg 0 .
\end{array}
$$

Definition 9 (see [31]). An element $(g, h) \in G \times G$ is called coupled fixed point of a mapping $\ddot{B}: G \times G \longrightarrow G$ if

$$
\begin{aligned}
& \ddot{B}(g, h)=g, \\
& \ddot{B}(h, g)=h .
\end{aligned}
$$

Now, in the following main results, we shall prove some unique coupled FP theorems depending on another function which is continuous, one-one, and subsequently convergent in FCM-spaces. We present some illustrative examples in support of our results. As a further study, we shall present two Volterra integral equations to ensure the existence of common solution to support our work.

\section{Main Results}

Now, we are in the position to present our first main result.

Theorem 1. Let $\ddot{B}: G \times G \longrightarrow G$ be a mapping in a complete FCM-space $\left(G, M_{c}, *\right)$ in which $M_{c}$ is triangular and $A$ is a continuous, one-one, and subsequently convergent selfmapping on $G$, that is, $\dot{A}: G \longrightarrow G$, satisfying

$$
\begin{aligned}
\frac{1}{M_{c}(\dot{A} \ddot{B}(g, h), \dot{A} \ddot{B}(\xi, \eta), t)}-1 \leq & \alpha\left(\frac{1}{M_{c}(\dot{A} g, \dot{A} \xi, t)}-1\right) \\
& +\beta\left[\left(\frac{1}{M_{c}(\dot{A} g, \dot{A} \ddot{B}(g, h), t)}-1\right)+\left(\frac{1}{M_{c}(\dot{A} \xi, \dot{A} \ddot{B}(\xi, \eta), t)}-1\right)\right] \\
& +\gamma\left[\left(\frac{1}{M_{c}(\dot{A} g, \dot{A} \ddot{B}(\xi, \eta), t)}-1\right)+\left(\frac{1}{M_{c}(\dot{A} \xi, \dot{A} \ddot{B}(g, h), t)}-1\right)\right],
\end{aligned}
$$

for all $g, h, \xi, \eta \in G, \quad t \gg 0$, and $\alpha, \beta, \gamma \in[0,1]$ with $\alpha+2 \beta+2 \gamma<1$. Then $\ddot{B}$ has a unique coupled FP. Also, if $\dot{A}$ converges sequently, then for every $g_{0} \in G$ the iterative sequence $\left\{\ddot{B}^{\ell} g_{o}\right\}$ converges to this coupled FP.

Proof. Consider any $g_{0}, h_{0} \in G$; we define sequences $\left\{g_{\ell}\right\}$ and $\left\{h_{\ell}\right\}$ in $\mathrm{G}$ such that

$$
\begin{aligned}
& \ddot{B}\left(g_{\ell}, h_{\ell}\right)=g_{\ell+1}, \\
& \ddot{B}\left(h_{\ell}, g_{\ell}\right)=h_{\ell+1}, \quad \text { for } \ell \geq 0 .
\end{aligned}
$$

$$
\begin{aligned}
\frac{1}{M_{c}\left(\dot{A} g_{\ell}, \dot{A} g_{\ell+1}, t\right)}-1= & \frac{1}{M_{c}\left(\dot{A} \ddot{B}\left(g_{\ell-1}, h_{\ell-1}\right), \dot{A} \ddot{B}\left(g_{\ell}, h_{\ell}\right), t\right)}-1 \\
\leq & \alpha\left(\frac{1}{M_{c}\left(\dot{A} g_{\ell-1}, \dot{A} g_{\ell}, t\right)}-1\right) \\
& +\beta\left[\left(\frac{1}{M_{c}\left(\dot{A} g_{\ell-1}, \dot{A} \ddot{B}\left(g_{\ell-1}, h_{\ell-1}\right), t\right)}-1\right)+\left(\frac{1}{M_{c}\left(\dot{A} g_{\ell}, \dot{A} \ddot{B}\left(g_{\ell}, h_{\ell}\right), t\right)}-1\right)\right]
\end{aligned}
$$




$$
\begin{aligned}
& +\gamma\left[\left(\frac{1}{M_{c}\left(\dot{A} g_{\ell-1}, \dot{A} \ddot{B}\left(g_{\ell}, h_{\ell}\right), t\right)}-1\right)+\left(\frac{1}{M_{c}\left(\dot{A} g_{\ell}, \dot{A} \ddot{B}\left(g_{\ell-1}, h_{\ell-1}\right), t\right)}-1\right)\right] \\
= & \alpha\left(\frac{1}{M_{c}\left(\dot{A} g_{\ell-1}, \dot{A} g_{\ell}, t\right)}-1\right) \\
& +\beta\left[\left(\frac{1}{M_{c}\left(\dot{A} g_{\ell-1}, \dot{A} g_{\ell}, t\right)}-1\right)+\left(\frac{1}{M_{c}\left(\dot{A} g_{\ell}, \dot{A} g_{\ell+1}, t\right)}-1\right)\right] \\
& +\gamma\left[\left(\frac{1}{M_{c}\left(\dot{A} g_{\ell-1}, \dot{A} g_{\ell+1}, t\right)}-1\right)+\left(\frac{1}{M_{c}\left(\dot{A} g_{\ell}, \dot{A} g_{\ell}, t\right)}-1\right)\right] \\
\leq & \alpha\left(\frac{1}{M_{c}\left(\dot{A} g_{\ell-1}, \dot{A} g_{\ell}, t\right)}-1\right) \\
& +\beta\left[\left(\frac{1}{M_{c}\left(\dot{A} g_{\ell-1}, \dot{A} g_{\ell}, t\right)}-1\right)+\left(\frac{1}{M_{c}\left(\dot{A} g_{\ell}, \dot{A} g_{\ell+1}, t\right)}-1\right)\right] \\
& +\gamma\left[\left(\frac{1}{M_{c}\left(\dot{A} g_{\ell-1}, \dot{A} g_{\ell}, t\right)}-1\right)+\left(\frac{1}{M_{c}\left(\dot{A} g_{\ell}, \dot{A} g_{\ell+1}, t\right)}-1\right)\right] .
\end{aligned}
$$

After simplification, we get that

$$
\frac{1}{M_{c}\left(\dot{A} g_{\ell}, \dot{A} g_{\ell+1}, t\right)}-1 \leq \theta\left(\frac{1}{M_{c}\left(\dot{A} g_{\ell-1}, \dot{A} g_{\ell}, t\right)}-1\right) \text {, }
$$$$
\frac{1}{M_{c}\left(\dot{A} g_{\ell-1}, \dot{A} g_{\ell}, t\right)}-1 \leq \theta\left(\frac{1}{M_{c}\left(\dot{A} g_{\ell-2}, \dot{A} g_{\ell-1}, t\right)}-1\right) \text {, }
$$

where $\theta$ is the same as in (8). Now, from (8) and (9) and by where $\theta=(\alpha+\beta+\gamma) /(1-\beta-\gamma)<1$. Similarly, from (5), induction, for $t \gg 0$, we have that

for $t \gg 0$, we have

$$
\begin{aligned}
\frac{1}{M_{c}\left(\dot{A} g_{\ell}, \dot{A} g_{\ell+1}, t\right)}-1 & \leq \theta\left(\frac{1}{M_{c}\left(\dot{A} g_{\ell-1}, \dot{A} g_{\ell}, t\right)}-1\right) \\
& \leq \theta^{2}\left(\frac{1}{M_{c}\left(\dot{A} g_{\ell-2}, \dot{A} g_{\ell-1}, t\right)}-1\right) \\
& \leq \cdots \leq \theta^{\ell}\left(\frac{1}{M_{c}\left(\dot{A} g_{0}, \dot{A} g_{1}, t\right)}-1\right) \longrightarrow 0, \quad \text { as } \ell \longrightarrow \infty
\end{aligned}
$$

It is shown that $\left\{\dot{A} g_{\ell}\right\}$ is a fuzzy cone contractive sequence; therefore,

$$
\lim _{\ell \longrightarrow \infty} M_{c}\left(\dot{A} g_{\ell}, \dot{A} g_{\ell+1}, t\right)=1, \quad \text { for } t \gg 0
$$


Now, for $j>\ell$ and for $t \gg 0$, we have

$$
\begin{aligned}
& \frac{1}{M_{c}\left(\dot{A} g_{\ell}, \dot{A} g_{j}, t\right)}-1 \\
& \leq\left(\frac{1}{M_{c}\left(\dot{A} g_{\ell}, \dot{A} g_{\ell+1}, t\right)}-1\right)+\left(\frac{1}{M_{c}\left(\dot{A} g_{\ell+1}, \dot{A} g_{\ell+2}, t\right)}-1\right)+\cdots+\left(\frac{1}{M_{c}\left(\dot{A} g_{j-1}, \dot{A} g_{j}, t\right)}-1\right) \\
& \leq \theta^{\ell}\left(\frac{1}{M_{c}\left(\dot{A} g_{o}, \dot{A} g_{1}, t\right)}-1\right)+\theta^{\ell+1}\left(\frac{1}{M_{c}\left(\dot{A} g_{0}, \dot{A} g_{1}, t\right)}-1\right)+\cdots+\theta^{j-1}\left(\frac{1}{M_{c}\left(\dot{A} g_{0}, \dot{A} g_{1}, t\right)}-1\right) \\
& =\left(\theta^{\ell}+\theta^{\ell+1}+\cdots+\theta^{j-1}\right)\left(\frac{1}{M_{c}\left(\dot{A} g_{0}, \dot{A} g_{1}, t\right)}-1\right) \\
& =\frac{\theta^{\ell}}{1-\theta}\left(\frac{1}{M_{c}\left(\dot{A} g_{o}, \dot{A} g_{1}, t\right)}-1\right) \longrightarrow 0, \quad \text { as } \ell \longrightarrow \infty .
\end{aligned}
$$

Hence, proving that $\left\{\dot{A} g_{\ell}\right\}$ is a Cauchy sequence, we have that

$$
\lim _{\ell, j \rightarrow \infty} M_{c}\left(\dot{A} g_{\ell}, \dot{A} g_{j}, t\right)=1, \quad \text { for } t \gg 0 .
$$

Now, for sequence $\left\{h_{\ell}\right\}_{\ell \geq 0}$ and from (5), for $t \gg 0$, we have

$$
\begin{aligned}
& \frac{1}{M_{c}\left(\dot{A} h_{\ell}, \dot{A} h_{\ell+1}, t\right)}-1=\frac{1}{M_{c}\left(\dot{A} \ddot{B}\left(h_{\ell-1}, g_{\ell-1}\right), \dot{A} \ddot{B}\left(h_{\ell}, g_{\ell}\right), t\right)}-1 \\
& \leq \alpha\left(\frac{1}{M_{c}\left(\dot{A} h_{\ell-1}, \dot{A} h_{\ell}, t\right)}-1\right) \\
& +\beta\left[\left(\frac{1}{M_{c}\left(\dot{A} h_{\ell-1}, \dot{A} \ddot{B}\left(h_{\ell-1}, g_{\ell-1}\right), t\right)}-1\right)+\left(\frac{1}{M_{c}\left(\dot{A} h_{\ell}, \dot{A} \ddot{B}\left(h_{\ell}, g_{\ell}\right), t\right)}-1\right)\right] \\
& +\gamma\left[\left(\frac{1}{M_{c}\left(\dot{A} h_{\ell-1}, \dot{A} \ddot{B}\left(h_{\ell}, g_{\ell}\right), t\right)}-1\right)+\left(\frac{1}{M_{c}\left(\dot{A} h_{\ell}, \dot{A} \ddot{B}\left(h_{\ell-1}, g_{\ell-1}\right), t\right)}-1\right)\right] \\
& =\alpha\left(\frac{1}{M_{c}\left(\dot{A} h_{\ell-1}, \dot{A} h_{\ell}, t\right)}-1\right) \\
& +\beta\left[\left(\frac{1}{M_{c}\left(\dot{A} h_{\ell-1}, \dot{A} h_{\ell}, t\right)}-1\right)+\left(\frac{1}{M_{c}\left(\dot{A} h_{\ell}, \dot{A} h_{\ell+1}, t\right)}-1\right)\right] \\
& +\gamma\left[\left(\frac{1}{M_{c}\left(\dot{A} h_{\ell-1}, \dot{A} h_{\ell+1}, t\right)}-1\right)+\left(\frac{1}{M_{c}\left(\dot{A} h_{\ell}, \dot{A} h_{\ell}, t\right)}-1\right)\right] \\
& \leq \alpha\left(\frac{1}{M_{c}\left(\dot{A} h_{\ell-1}, \dot{A} h_{\ell}, t\right)}-1\right) \\
& +\beta\left[\left(\frac{1}{M_{c}\left(\dot{A} h_{\ell-1}, \dot{A} h_{\ell}, t\right)}-1\right)+\left(\frac{1}{M_{c}\left(\dot{A} h_{\ell}, \dot{A} h_{\ell+1}, t\right)}-1\right)\right] \\
& +\gamma\left[\left(\frac{1}{M_{c}\left(\dot{A} h_{\ell-1}, \dot{A} h_{\ell}, t\right)}-1\right)+\left(\frac{1}{M_{c}\left(\dot{A} h_{\ell}, \dot{A} h_{\ell+1}, t\right)}-1\right)\right] \text {. }
\end{aligned}
$$


After simplification, we get that

$$
\frac{1}{M_{c}\left(\dot{A} h_{\ell}, \dot{A} h_{\ell+1}, t\right)}-1 \leq \theta\left(\frac{1}{M_{c}\left(\dot{A} h_{\ell-1}, \dot{A} h_{\ell}, t\right)}-1\right)
$$$$
\frac{1}{M_{c}\left(\dot{A} h_{\ell-1}, \dot{A} h_{\ell}, t\right)}-1 \leq \theta\left(\frac{1}{M_{c}\left(\dot{A} h_{\ell-2}, \dot{A} h_{\ell-1}, t\right)}-1\right) \text {, }
$$

for $t \gg 0$,

where $\theta$ is the same as in (8). Similarly, from (5) for $t \gg 0$, we where $\theta$ is the same as in (8). Now, from (15) and (16) and by induction, for $t \gg 0$, we have that have

$$
\begin{aligned}
\frac{1}{M_{c}\left(\dot{A} h_{\ell}, \dot{A} h_{\ell+1}, t\right)}-1 & \leq \theta\left(\frac{1}{M_{c}\left(\dot{A} h_{\ell-1}, \dot{A} h_{\ell}, t\right)}-1\right) \\
& \leq \theta^{2}\left(\frac{1}{M_{c}\left(\dot{A} h_{\ell-2}, \dot{A} h_{\ell-1}, t\right)}-1\right) \\
& \leq \cdots \leq \theta^{\ell}\left(\frac{1}{M_{c}\left(\dot{A} h_{0}, \dot{A} h_{1}, t\right)}-1\right) \rightarrow 0, \quad \text { as } \ell \longrightarrow \infty
\end{aligned}
$$

It is shown that $\left\{\dot{A} h_{\ell}\right\}$ is a fuzzy cone contractive sequence.

$$
\lim _{\ell \longrightarrow \infty} M_{c}\left(\dot{A} h_{\ell}, \dot{A} h_{\ell+1}, t\right)=1, \quad \text { for } t \gg 0 .
$$

$$
\begin{aligned}
& \frac{1}{M_{c}\left(\dot{A} h_{\ell}, \dot{A} h_{j}, t\right)}-1 \\
& \leq\left(\frac{1}{M_{c}\left(\dot{A} h_{\ell}, \dot{A} h_{\ell+1}, t\right)}-1\right)+\left(\frac{1}{M_{c}\left(\dot{A} h_{\ell+1}, \dot{A} h_{\ell+2}, t\right)}-1\right)+\cdots+\left(\frac{1}{M_{c}\left(\dot{A} h_{j-1}, \dot{A} h_{j}, t\right)}-1\right) \\
& \leq \theta^{\ell}\left(\frac{1}{M_{c}\left(\dot{A} h_{o}, \dot{A} h_{1}, t\right)}-1\right)+\theta^{\ell+1}\left(\frac{1}{M_{c}\left(\dot{A} h_{0}, \dot{A} h_{1}, t\right)}-1\right)+\cdots+\theta^{j-1}\left(\frac{1}{M_{c}\left(\dot{A} h_{0}, \dot{A} h_{1}, t\right)}-1\right) \\
& =\left(\theta^{\ell}+\theta^{\ell+1}+\cdots+\theta^{j-1}\right)\left(\frac{1}{M_{c}\left(\dot{A} h_{0}, \dot{A} h_{1}, t\right)}-1\right) \\
& =\frac{\theta^{\ell}}{1-\theta}\left(\frac{1}{M_{c}\left(\dot{A} h_{0}, \dot{A} h_{1}, t\right)}-1\right) \longrightarrow 0, \text { as } \longrightarrow \infty .
\end{aligned}
$$

Hence, proving that $\left\{\dot{A} h_{\ell}\right\}_{\ell \geq 0}$ is a Cauchy sequence, we have that

$$
\lim _{\ell, j \rightarrow \infty} M_{c}\left(\dot{A} h_{\ell}, \dot{A} h_{j}, t\right)=1, \quad \text { for } t \gg 0 .
$$

Since $G$ is complete, $\left\{\dot{A} g_{\ell}\right\}$ and $\left\{\dot{A} h_{\ell}\right\}$ are Cauchy sequences in $G$; therefore, $\dot{A} g_{\ell} \longrightarrow g \in G$ and
$\dot{A} h_{\ell} \longrightarrow h \in G$ as $\ell \longrightarrow \infty$; that is, $\lim _{\ell \longrightarrow \infty} \dot{A} g_{\ell}=g$ and $\lim _{\ell \rightarrow \infty} \dot{A} h_{\ell}=h$. Since $\dot{A}$ is subsequently convergent, $\left\{g_{\ell}\right\}$ has a convergent subsequence. So there exist $g \in G$ and $\left\{g_{\ell(k)}\right\}$ in $\mathrm{G}$ such that $\lim _{k \longrightarrow \infty} g_{\ell(k)}=g$. Since $\dot{A}$ is continuous, $\lim _{k \longrightarrow \infty} g_{\ell(k)}=g$, and $\lim _{k \longrightarrow \infty} \dot{A} g_{n(k)}=\dot{A} g$. Now, from (5), for $t \gg 0$, we have 


$$
\begin{aligned}
\frac{1}{M_{c}(\dot{A} \ddot{B}(g, h), \dot{A} g, t)}-1 \leq & \left(\frac{1}{M_{c}\left(\dot{A} \ddot{B}(g, h), \dot{A} \ddot{B}\left(g_{\ell-1}, h_{\ell-1}\right), t\right)}-1\right)+\left(\frac{1}{M_{c}\left(\dot{A} \ddot{B}\left(g_{\ell-1}, h_{\ell-1}\right), \dot{A} \ddot{B}\left(g_{\ell}, h_{\ell}\right), t\right)}-1\right) \\
& +\left(\frac{1}{M_{c}\left(\dot{A} \ddot{B}\left(g_{\ell}, h_{\ell}\right), \dot{A} g, t\right)}-1\right) \leq \alpha\left(\frac{1}{M_{c}\left(\dot{A} g, \dot{A} g_{\ell-1}, t\right)}-1\right) \\
+ & \beta\left[\left(\frac{1}{M_{c}(\dot{A} g, \dot{A} \ddot{B}(g, h), t)}-1\right)+\left(\frac{1}{M_{c}\left(\dot{A} g_{\ell-1}, \dot{A} \ddot{B}\left(g_{\ell-1}, h_{\ell-1}\right), t\right)}-1\right)\right] \\
+ & \gamma\left[\left(\frac{1}{M_{c}\left(\dot{A} g, \dot{A} \ddot{B}\left(g_{\ell-1}, h_{\ell-1}\right), t\right)}-1\right)+\left(\frac{1}{M_{c}\left(\dot{A} g_{\ell-1}, \dot{A} \ddot{B}\left(g_{\ell}, h_{\ell}\right), t\right)}-1\right)\right] \\
+ & \theta^{\ell}\left(\frac{1}{M_{c}\left(\dot{A} g_{0}, \dot{A} g_{1}, t\right)}-1\right)+\left(\frac{1}{M_{c}\left(\dot{A} \ddot{B}\left(g_{\ell}, h_{\ell}\right), \dot{A} g, t\right)}-1\right) .
\end{aligned}
$$

After simplification, for $t \gg 0$, we have

$$
\begin{aligned}
\frac{1}{M_{c}(\dot{A} \ddot{B}(g, h), \dot{A} g, t)}-1 \leq & \frac{\alpha}{1-\beta}\left(\frac{1}{M\left(\dot{A} g, \dot{A} g_{\ell-1}, t\right)}-1\right)+\frac{\beta}{1-\beta}\left(\frac{1}{M_{c}\left(\dot{A} g_{\ell-1}, \dot{A} \ddot{B}\left(g_{\ell-1}, h_{\ell-1}\right), t\right)}-1\right) \\
& +\frac{\gamma}{1-\beta}\left[\left(\frac{1}{M_{c}\left(\dot{A} g, \dot{A} \ddot{B}\left(g_{\ell-1}, h_{\ell-1}\right), t\right)}-1\right)+\left(\frac{1}{M_{c}\left(\dot{A} g_{\ell-1}, \dot{A} \ddot{B}\left(g_{\ell}, h_{\ell}\right), t\right)}-1\right)\right] \\
& +\frac{1}{1-\beta} \theta^{\ell}\left(\frac{1}{M_{c}\left(\dot{A} g_{0}, \dot{A} g_{1}, t\right)}-1\right)+\frac{1}{1-\beta}\left(\frac{1}{M_{c}\left(\dot{A} \ddot{B}\left(g_{\ell}, h_{\ell}\right), A g, t\right)}-1\right) \longrightarrow 0, \text { as } \ell \longrightarrow \infty
\end{aligned}
$$

Hence, we get that $M_{c}(\dot{A} \ddot{B}(g, h), \dot{A} g, t)=1$; this implies that $\dot{A} \ddot{B}(g, h)=\dot{A} g$. Since $\dot{A}$ is one-one, $\ddot{B}(g, h)=g$. Next, we have to prove that $\ddot{B}(h, g)=h$. Then, from (5), for $t \gg 0$, we have

$$
\begin{aligned}
\frac{1}{M_{c}(\dot{A} \ddot{B}(h, g), \dot{A} h, t)}-1 \leq & \left(\frac{1}{M_{c}\left(\dot{A} \ddot{B}(h, g), \dot{A} \ddot{B}\left(h_{\ell-1}, g_{\ell-1}\right), t\right)}-1\right)+\left(\frac{1}{M_{c}\left(\dot{A} \ddot{B}\left(h_{\ell-1}, g_{\ell-1}\right), \dot{A} \ddot{B}\left(h_{\ell}, g_{\ell}\right), t\right)}-1\right) \\
& +\left(\frac{1}{M_{c}\left(\dot{A} \ddot{B}\left(h_{\ell}, g_{\ell}\right), \dot{A} h, t\right)}-1\right) \leq \alpha\left(\frac{1}{M_{c}\left(\dot{A} h, \dot{A} h_{\ell-1}, t\right)}-1\right) \\
& +\beta\left[\left(\frac{1}{M_{c}(\dot{A} h, \dot{A} \ddot{B}(h, g), t)}-1\right)+\left(\frac{1}{M_{c}\left(\dot{A} h_{\ell-1}, \dot{A} \ddot{B}\left(h_{\ell-1}, g_{\ell-1}\right), t\right)}-1\right)\right] \\
& +\gamma\left[\left(\frac{1}{M_{c}\left(\dot{A} h, \dot{A} \ddot{B}\left(h_{\ell-1}, g_{\ell-1}\right), t\right)}-1\right)+\left(\frac{1}{M_{c}\left(\dot{A} h_{\ell-1}, \dot{A} \ddot{B}\left(h_{\ell}, g_{\ell}\right), t\right)}-1\right)\right] \\
& +\theta^{\ell}\left(\frac{1}{M_{c}\left(\dot{A} h_{0}, \dot{A} h_{1}, t\right)}-1\right)+\left(\frac{1}{M_{c}\left(\dot{A} \ddot{B}\left(h_{\ell}, g_{\ell}\right), \dot{A} h, t\right)}-1\right) .
\end{aligned}
$$


After simplification, for $t \gg 0$, we have

$$
\begin{aligned}
\frac{1}{M_{c}(\dot{A} \ddot{B}(h, g), \dot{A} h, t)}-1 \leq & \frac{\alpha}{1-\beta}\left(\frac{1}{M_{c}\left(\dot{A} h, \dot{A} h_{\ell-1}, t\right)}-1\right)+\frac{\beta}{1-\beta}\left(\frac{1}{M_{c}\left(\dot{A} h_{\ell-1}, \dot{A} \ddot{B}\left(h_{\ell-1}, g_{\ell-1}\right), t\right)}-1\right) \\
& +\frac{\gamma}{1-\beta}\left[\left(\frac{1}{M_{c}\left(\dot{A} h, \dot{A} \ddot{B}\left(h_{\ell-1}, g_{\ell-1}\right), t\right)}-1\right)+\left(\frac{1}{M_{c}\left(\dot{A} h_{\ell-1}, \dot{A} \ddot{B}\left(h_{\ell}, g_{\ell}\right), t\right)}-1\right)\right] \\
& +\frac{1}{1-\beta} \theta^{\ell}\left(\frac{1}{M_{c}\left(\dot{A} h_{0}, \dot{A} h_{1}, t\right)}-1\right)+\frac{1}{1-\beta}\left(\frac{1}{M_{c}\left(\dot{A} \ddot{B}\left(h_{\ell}, g_{\ell}\right), \dot{A} g, t\right)}-1\right) \longrightarrow 0, \text { as } \ell \longrightarrow \infty .
\end{aligned}
$$

Hence, we get that $M_{c}(\dot{A} \ddot{B}(h, g), \dot{A} h, t)=1$, for $t \gg 0$; this implies that $\dot{A} \ddot{B}(h, g)=\dot{A} h$. Since $\dot{A}$ is one-one, we get $\ddot{B}(h, g)=h$.
For uniqueness, suppose that $\left(g_{1}, h_{1}\right)$ and $\left(h_{1}, g_{1}\right)$ are coupled fixed-point pairs in $G \times G$ such that $\ddot{B}\left(g_{1}, h_{1}\right)=g_{1}$ and $\ddot{B}\left(h_{1}, g_{1}\right)=h_{1}$. Now, from (5), for $t \gg 0$, we have

$$
\begin{aligned}
& \frac{1}{M_{c}\left(\dot{A} g, \dot{A} g_{1}, t\right)}-1=\frac{1}{M_{c}\left(\dot{A} \ddot{B}(g, h), \dot{A} \ddot{B}\left(g_{1}, h_{1}\right), t\right)}-1 \\
& \leq \alpha\left(\frac{1}{M_{c}\left(\dot{A} g, \dot{A} g_{1}, t\right)}-1\right) \\
& +\beta\left[\left(\frac{1}{M_{c}(\dot{A} g, \dot{A} \ddot{B}(g, h), t)}-1\right)+\left(\frac{1}{M_{c}\left(\dot{A} g_{1}, \dot{A} \ddot{B}\left(g_{1}, h_{1}\right), t\right)}-1\right)\right] \\
& +\gamma\left[\left(\frac{1}{M_{c}\left(\dot{A} g, \dot{A} \ddot{B}\left(g_{1}, h_{1}\right), t\right)}-1\right)+\left(\frac{1}{M_{c}\left(\dot{A} g_{1}, \dot{A} \ddot{B}(g, h), t\right)}-1\right)\right] \\
& =(\alpha+2 \gamma)\left(\frac{1}{M_{c}\left(\dot{A} g, \dot{A} g_{1}, t\right)}-1\right) \\
& =(\alpha+2 \gamma)\left(\frac{1}{M_{c}\left(\dot{A} \ddot{B}(g, h), \dot{A} \ddot{B}\left(g_{1}, h_{1}\right), t\right)}-1\right) \\
& \leq(\alpha+2 \gamma)^{2}\left(\frac{1}{M_{c}\left(\dot{A} g, \dot{A} g_{1}, t\right)}-1\right) \\
& \leq \cdots \leq(\alpha+2 \gamma)^{\ell}\left(\frac{1}{M_{c}\left(\dot{A} g, \dot{A} g_{1}, t\right)}-1\right) \longrightarrow 0, \quad \text { as } \ell \longrightarrow \infty
\end{aligned}
$$


Hence, we get that $M_{c}\left(\dot{A} g, \dot{A} g_{1}, t\right)=1$ for $t \gg 0$; this implies that $g=g_{1}$. Similarly, again from (5), for $t \gg 0$, we have

$$
\begin{aligned}
\frac{1}{M_{c}\left(\dot{A} h, \dot{A} h_{1}, t\right)}-1= & \frac{1}{M_{c}\left(\dot{A} \ddot{B}(h, g), \dot{A} \ddot{B}\left(h_{1}, g_{1}\right), t\right)}-1 \\
\leq & \alpha\left(\frac{1}{M_{c}\left(\dot{A} h, \dot{A} h_{1}, t\right)}-1\right) \\
& +\beta\left[\left(\frac{1}{M_{c}(\dot{A} h, \dot{A} \ddot{B}(h, g), t)}-1\right)+\left(\frac{1}{M_{c}\left(\dot{A} h_{1}, \dot{A} \ddot{B}\left(h_{1}, g_{1}\right), t\right)}-1\right)\right] \\
& +\gamma\left[\left(\frac{1}{M_{c}\left(\dot{A} h, \dot{A} \ddot{B}\left(h_{1}, g_{1}\right), t\right)}-1\right)+\left(\frac{1}{M_{c}\left(\dot{A} h_{1}, \dot{A} \ddot{B}(h, g), t\right)}-1\right)\right] \\
= & (\alpha+2 \gamma)\left(\frac{1}{M_{c}\left(\dot{A} h, \dot{A} h_{1}, t\right)}-1\right) \\
= & (\alpha+2 \gamma)\left(\frac{1}{M_{c}\left(\dot{A} \ddot{B}(h, g), \dot{A} \ddot{B}\left(h_{1}, g_{1}\right), t\right)}-1\right) \\
\leq & (\alpha+2 \gamma)^{2}\left(\frac{1}{M_{c}\left(\dot{A} h, \dot{A} h_{1}, t\right)}-1\right) \\
\leq & \cdots \leq(\alpha+2 \gamma)^{\ell}\left(\frac{1}{M_{c}\left(\dot{A} h, \dot{A} h_{1}, t\right)}-1\right) \longrightarrow 0, \quad \text { as } \ell \longrightarrow \infty .
\end{aligned}
$$

Hence, we get that $M_{c}\left(\dot{A} h, \dot{A} h_{1}, t\right)=1$ for $t \gg 0$, and this implies that $h=h_{1}$.

Corollary 1. Let $\ddot{B}: G \times G \longrightarrow G$ be a mapping in a complete FCM-space $\left(G, M_{c}, *\right)$ in which $M_{c}$ is triangular and $A$ is a continuous, one-one, and subsequently convergent selfmapping on $G$, that is, $\dot{A}: G \longrightarrow G$, satisfying

$$
\begin{aligned}
& \frac{1}{M_{c}(\dot{A} \ddot{B}(g, h), \dot{A} \ddot{B}(\xi, \eta), t)}-1 \leq \alpha\left(\frac{1}{M_{c}(\dot{A} g, \dot{A} \xi, t)}-1\right) \\
& +\beta\left[\left(\frac{1}{M_{c}(\dot{A} g, \dot{A} \ddot{B}(g, h), t)}-1\right)+\left(\frac{1}{M_{c}(\dot{A} \xi, \dot{A} \ddot{B}(\xi, \eta), t)}-1\right)\right],
\end{aligned}
$$

for all $g, h, \xi, \eta \in G, t \gg 0$, and $\alpha, \beta \in[0,1]$ with $\alpha+2 \beta<1$. Then $\ddot{B}$ has a unique coupled FP. Also, if $\dot{A}$ converges sequently, then, for every $g_{0} \in G$, the iterative sequence $\left\{\ddot{B}^{\ell} g_{o}\right\}$ converges to this coupled FP.

Corollary 2. Let $\ddot{B}: G \times G \longrightarrow G$ be a mapping in a complete FCM-space $\left(G, M_{c}, *\right)$ in which $M_{c}$ is triangular and $A$ is a continuous, one-one, and subsequently convergent selfmapping on $G$, that is, $\dot{A}: G \longrightarrow G$, satisfying

$$
\begin{aligned}
& \frac{1}{M_{c}(\dot{A} \ddot{B}(g, h), \dot{A} \ddot{B}(\xi, \eta), t)}-1 \leq \alpha\left(\frac{1}{M_{c}(\dot{A} g, \dot{A} \xi, t)}-1\right) \\
& +\gamma\left[\left(\frac{1}{M_{c}(\dot{A} g, \dot{A} \ddot{B}(\xi, \eta), t)}-1\right)+\left(\frac{1}{M_{c}(\dot{A} \xi, \dot{A} \ddot{B}(g, h), t)}-1\right)\right],
\end{aligned}
$$

for all $g, h, \xi, \eta \in G, t \gg 0$, and $\alpha, \gamma \in[0,1]$ with $\alpha+2 \gamma<1$. Then $\ddot{B}$ has a unique coupled FP. Also, if $\dot{A}$ converges sequently, then, for every $g_{0} \in G$, the iterative sequence $\left\{\ddot{B}^{\ell} g_{o}\right\}$ converges to this coupled FP.

If we use $\dot{A}$ as an identity self-mapping, that is, $\dot{A}=I$ in Theorem 1, then we get the following corollary.

Corollary 3. Let $\ddot{B}: G \times G \longrightarrow G$ be a mapping in a complete FCM-space $\left(G, M_{c}, *\right)$ in which $M_{c}$ is triangular, satisfying

$$
\begin{aligned}
& \frac{1}{M_{c}(\ddot{B}(g, h), \ddot{B}(\xi, \eta), t)}-1 \leq \alpha\left(\frac{1}{M_{c}(g, \xi, t)}-1\right) \\
& \quad+\beta\left[\left(\frac{1}{M_{c}(g, \ddot{B}(g, h), t)}-1\right)+\left(\frac{1}{M_{c}(\xi, \ddot{B}(\xi, \eta), t)}-1\right)\right] \\
& \quad+\gamma\left[\left(\frac{1}{M_{c}(g, \ddot{B}(\xi, \eta), t)}-1\right)+\left(\frac{1}{M_{c}(\xi, \ddot{B}(g, h), t)}-1\right)\right],
\end{aligned}
$$


for all $g, h, \xi, \eta \in G, \quad t \gg 0$, and $\alpha, \beta, \gamma \in[0,1]$ with $\alpha+2 \beta+2 \gamma<1$. Then $\ddot{B}$ has a unique coupled FP in $G$.

Example 1. Let $G=\{0\} \cup\{(1 / 2),(1 / 3),(1 / 4), \ldots\}$, and let a fuzzy metric $M_{c}: G^{2} \times(0, \infty) \longrightarrow[0,1]$ be defined by

$$
M_{c}(g, h, t)=\frac{t}{t+d(g, h)}, \quad \text { where } d(g, h)=|g-h|,
$$

$\forall g, h \in G$ and $t>0$. Then easily one can verify that $M_{c}$ is triangular and $\left(G, M_{c}, *\right)$ is a complete FCM-space. We define $\ddot{B}: G \times G \longrightarrow G$ by $\ddot{B}(0,0)=0$ and $\ddot{B}((1 / n),(1 / \ell))=$ $1 /(n+\ell+2)$ for all $n, \ell \geq 2$; and a mapping $\dot{A}: G \longrightarrow G$ is defined as $\dot{A}(0)=0$ and $\dot{A}(1 / n)=\left(1 / n^{n}\right)$. Then, by using (30), for $t \gg 0$, we have

$$
\begin{aligned}
& \frac{1}{M_{c}(\dot{A} \ddot{B}((1 / n),(1 / \ell)), \dot{A} \ddot{B}((1 / p),(1 / q)), t)}-1 \\
& =\frac{1}{t}\left[d\left(\dot{A} \ddot{B}\left(\frac{1}{n}, \frac{1}{\ell}\right), \dot{A} \ddot{B}\left(\frac{1}{p}, \frac{1}{q}\right)\right)\right] \\
& =\frac{1}{t}\left[\frac{1}{(n+\ell+2)^{n+\ell+2}}-\frac{1}{(p+q+2)^{p+q+2}}\right] .
\end{aligned}
$$

Let $\quad(1 / t)\left(1 /(n+\ell+2)^{n+\ell+2}\right) \leq(1 / 5 t)\left[\left(2 / n^{n}\right)-(1 /\right.$ $\left.\left.(n+\ell+2)^{n+\ell+2}\right)\right]$. Then, from (31), we have

$$
\begin{aligned}
& \frac{1}{t}\left.\frac{1}{(n+\ell+2)^{n+\ell+2}}-\frac{1}{(p+q+2)^{p+q+2}}\right] \\
& \leq \frac{1}{5 t}\left[\left(\frac{2}{n^{n}}-\frac{1}{(n+\ell+2)^{n+\ell+2}}\right)-\left(\frac{2}{p^{p}}-\frac{1}{(p+q+2)^{p+q+2}}\right)\right] \\
&= \frac{1}{t}\left[\frac{1}{5 n^{n}}+\frac{1}{5 n^{n}}-\frac{1}{10(n+\ell+2)^{n+\ell+2}}-\frac{1}{10(n+\ell+2)^{n+\ell+2}}\right] \\
&- \frac{1}{t}\left[\frac{1}{5 p^{p}}+\frac{1}{5 p^{p}}-\frac{1}{10(p+q+2)^{p+q+2}}-\frac{1}{10(p+q+2)^{p+q+2}}\right] \\
&= \frac{1}{t}\left(\frac{1}{5 n^{n}}-\frac{1}{5 p^{p}}\right)+\frac{1}{t}\left[\frac{1}{5 n^{n}}-\frac{1}{10(n+\ell+2)^{n+\ell+2}}-\frac{1}{10(n+\ell+2)^{n+\ell+2}}\right] \\
&-\frac{1}{t}\left[\frac{1}{5 p^{p}}-\frac{1}{10(p+q+2)^{p+q+2}}-\frac{1}{10(p+q+2)^{p+q+2}}\right] \\
&= \frac{1}{t}\left(\frac{1}{5 n^{n}}-\frac{1}{5 p^{p}}\right)+\frac{1}{t}\left[\left(\frac{1}{10 n^{n}}-\frac{1}{10(n+\ell+2)^{n+\ell+2}}\right)+\left(\frac{1}{10 p^{p}}-\frac{1}{10(p+q+2)^{p+q+2}}\right)\right] \\
&+\frac{1}{t}\left[\left(\frac{1}{10 n^{n}}-\frac{1}{10(p+q+2)^{p+q+2}}\right)+\left(\frac{1}{10 p^{p}}-\frac{1}{10(n+\ell+2)^{n+\ell+2}}\right)\right] \\
&= \frac{1}{5 t}\left(\frac{1}{n^{n}}-\frac{1}{p^{p}}\right)+\frac{1}{10 t}\left[\left(\frac{1}{n^{n}}-\frac{1}{(n+\ell+2)^{n+\ell+2}}\right)+\left(\frac{1}{p^{p}}-\frac{1}{(p+q+2)^{p+q+2}}\right)\right] \\
&\left.\left.+\frac{1}{(p+q+2)^{p+q+2}}\right)+\left(\frac{1}{p^{p}}-\frac{1}{(n+\ell+2)^{n+\ell+2}}\right)\right] . \\
&\left.n^{n}-\frac{1}{(1}\right)
\end{aligned}
$$

Hence, we get that

$$
\begin{aligned}
\frac{1}{M_{c}(\dot{A} \ddot{B}((1 / n),(1 / \ell)), \dot{A} \ddot{B}((1 / p),(1 / q)), t)}-1 \leq & \frac{1}{5}\left(\frac{1}{M_{c}(\dot{A}(1 / n), \dot{A}(1 / p), t)}-1\right) \\
& +\frac{1}{10}\left[\left(\frac{1}{M_{c}(\dot{A}(1 / n), \dot{A} \ddot{B}((1 / n),(1 / \ell)), t)}-1\right)+\left(\frac{1}{M_{c}(\dot{A}(1 / p), \dot{A} \ddot{B}((1 / p),(1 / q)), t)}-1\right)\right] \\
& +\frac{1}{10}\left[\left(\frac{1}{M_{c}(\dot{A}(1 / n), \dot{A} \ddot{B}((1 / p),(1 / q)), t)}-1\right)+\left(\frac{1}{M_{c}(\dot{A}(1 / p), \dot{A} \ddot{B}((1 / n),(1 / m)), t)}-1\right)\right] .
\end{aligned}
$$


Thus, inequality (33) satisfies all the conditions of Theorem 1 with $\alpha=(1 / 5)$ and $\beta=\gamma=(1 / 10)$ and $\ddot{B}$ has a unique coupled fixed point; that is, $\ddot{B}(0,0)=0$.
Theorem 2. Let $\ddot{B}: G \times G \longrightarrow G$ be a mapping in a complete FCM-space $\left(G, M_{c}, *\right)$ in which $M_{c}$ is triangular and $A$ is a continuous, one-one, and subsequently convergent selfmapping on $G$, that is, $\dot{A}: G \longrightarrow G$, satisfying

$$
\begin{aligned}
\frac{1}{M_{c}(\dot{A} \ddot{B}(g, h), \dot{A} \ddot{B}(\xi, \eta), t)}-1 \leq & \alpha\left(\frac{1}{M_{c}(\dot{A} g, \dot{A} \xi, t)}-1\right) \\
& +\beta\left[\left(\frac{1}{M_{c}(\dot{A} g, \dot{A} \ddot{B}(g, h), t)}-1\right)+\left(\frac{1}{M_{c}(\dot{A} \xi, \dot{A} \ddot{B}(\xi, \eta), t)}-1\right)\right] \\
& +\gamma\left[\left(\frac{1}{M_{c}(\dot{A} \xi, \dot{A} \ddot{B}(g, h), t) * M_{c}(\dot{A} \xi, \dot{A} \ddot{B}(\xi, \eta), t)}-1\right)\right],
\end{aligned}
$$

for all $g, h, \xi, \eta \in G, \quad t \gg 0$, and $a, b, c \in[0,1]$ with $\alpha+2 \beta+\gamma<1$. Then $\ddot{B}$ has a unique coupled FP. Also, if $\dot{A}$ converges sequently, then, for each $g_{0} \in G$, the iterative sequence $\left\{\ddot{B}^{\ell} g_{0}\right\}$ converges to this coupled FP.

Proof. Let any $g_{0}, h_{0} \in G$, and we define sequence $\left\{g_{\ell}\right\}$ by

$$
\begin{aligned}
& \ddot{B}\left(g_{\ell}, h_{\ell}\right)=g_{\ell+1}, \\
& \ddot{B}\left(h_{\ell}, g_{\ell}\right)=h_{\ell+1}, \quad \text { for } \ell \geq 0 .
\end{aligned}
$$

Now, from (34), for $t \gg 0$, we have

$$
\begin{aligned}
& \frac{1}{M_{c}\left(\dot{A} g_{\ell}, \dot{A} g_{\ell+1}, t\right)}-1=\frac{1}{M_{c}\left(\dot{A} \ddot{B}\left(g_{\ell-1}, h_{\ell-1}\right), \dot{A} \ddot{B}\left(g_{\ell}, h_{\ell}\right), t\right)}-1 \\
& \leq \alpha\left(\frac{1}{M_{c}\left(\dot{A} g_{\ell-1}, \dot{A} g_{\ell}, t\right)}-1\right) \\
& +\beta\left[\left(\frac{1}{M_{c}\left(\dot{A} g_{\ell-1}, \dot{A} \ddot{B}\left(g_{\ell-1}, h_{\ell-1}\right), t\right)}-1\right)+\left(\frac{1}{M_{c}\left(\dot{A} g_{\ell}, \dot{A} \ddot{B}\left(g_{\ell}, h_{\ell}\right), t\right)}-1\right)\right] \\
& +\gamma\left(\frac{1}{M_{c}\left(\dot{A} g_{\ell}, \dot{A} \ddot{B}\left(g_{\ell-1}, h_{\ell-1}\right), t\right) * M_{c}\left(\dot{A} g_{\ell}, \dot{A} \ddot{B}\left(g_{\ell}, h_{\ell}\right), t\right)}-1\right) \\
& =\alpha\left(\frac{1}{M_{c}\left(\dot{A} g_{\ell-1}, \dot{A} g_{\ell}, t\right)}-1\right) \\
& +\beta\left[\left(\frac{1}{M_{c}\left(\dot{A} g_{\ell-1}, \dot{A} g_{\ell}, t\right)}-1\right)+\left(\frac{1}{M_{c}\left(\dot{A} g_{\ell}, \dot{A} g_{\ell+1}, t\right)}-1\right)\right] \\
& +\gamma\left(\frac{1}{M_{c}\left(\dot{A} g_{\ell}, \dot{A} g_{\ell}, t\right) * M_{c}\left(\dot{A} g_{\ell}, \dot{A} g_{\ell+1}, t\right)}-1\right) \\
& =\alpha\left(\frac{1}{M_{c}\left(\dot{A} g_{\ell-1}, \dot{A} g_{\ell}, t\right)}-1\right) \\
& +\beta\left[\left(\frac{1}{\left.M_{c}\left(\dot{A} g_{\ell-1}\right), \dot{A} g_{\ell}, t\right)}-1\right)+\left(\frac{1}{M_{c}\left(\dot{A} g_{\ell}, \dot{A} g_{\ell+1}, t\right)}-1\right)\right] \\
& +\gamma\left(\frac{1}{M_{c}\left(\dot{A} g_{\ell}, \dot{A} g_{\ell+1}, t\right)}-1\right) .
\end{aligned}
$$


After simplification, we get that

$$
\begin{array}{r}
\frac{1}{M_{c}\left(\dot{A} g_{\ell}, \dot{A} g_{\ell+1}, t\right)}-1 \leq \eta\left(\frac{1}{M_{c}\left(\dot{A} g_{\ell-1}, \dot{A} g_{\ell}, t\right)}-1\right) \\
\text { for } t \gg 0,
\end{array}
$$$$
\frac{1}{M_{c}\left(\dot{A} g_{\ell-1}, \dot{A} g_{\ell}, t\right)}-1 \leq \eta\left(\frac{1}{M_{c}\left(\dot{A} g_{\ell-2}, \dot{A} g_{\ell-1}, t\right)}-1\right),
$$$$
\text { for } t \gg 0 \text {, }
$$

where $\eta=(\alpha+\beta) /(1-\beta-\gamma)<1$. Similarly, again from

where $\eta$ is the same as in (37). Now, from (37) and (38) and by induction, for $t \gg 0$, we have

(34), for $t \gg 0$, we have

$$
\begin{aligned}
\frac{1}{M_{c}\left(\dot{A} g_{\ell}, \dot{A} g_{\ell+1}, t\right)}-1 & \leq \eta\left(\frac{1}{M_{c}\left(\dot{A} g_{\ell-1}, \dot{A} g_{\ell}, t\right)}-1\right) \\
& \leq \eta^{2}\left(\frac{1}{M_{c}\left(\dot{A} g_{\ell-2}, \dot{A} g_{\ell-1}, t\right)}-1\right) \\
& \leq \cdots \leq \eta^{\ell}\left(\frac{1}{M_{c}\left(\dot{A} g_{o}, \dot{A} g_{1}, t\right)}-1\right) \longrightarrow 0, \quad \text { as } \ell \longrightarrow \infty
\end{aligned}
$$

Hence, we get that $\left\{\dot{A} g_{\ell}\right\}_{\ell \geq 0}$ is a fuzzy cone contractive sequence; therefore,

$$
\lim _{\ell \longrightarrow \infty} M_{c}\left(\dot{A} g_{\ell}, \dot{A} g_{\ell+1}, t\right)=1, \quad t \gg 0 .
$$

Now, for $j>\ell$ and for $t \gg 0$, we have

$$
\begin{aligned}
& \frac{1}{M_{c}\left(\dot{A} g_{\ell}, \dot{A} g_{j}, t\right)}-1 \\
& \leq\left(\frac{1}{M_{c}\left(\dot{A} g_{\ell}, \dot{A} g_{\ell+1}, t\right)}-1\right)+\left(\frac{1}{M_{c}\left(\dot{A} g_{\ell+1}, \dot{A} g_{\ell+2}, t\right)}-1\right)+\cdots+\left(\frac{1}{M_{c}\left(\dot{A} g_{j-1}, \dot{A} g_{j}, t\right)}-1\right) \\
& \leq \eta^{\ell}\left(\frac{1}{M_{c}\left(\dot{A} g_{o}, \dot{A} g_{1}, t\right)}-1\right)+\eta^{\ell+1}\left(\frac{1}{M_{c}\left(\dot{A} g_{o}, \dot{A} g_{1}, t\right)}-1\right)+\cdots+\eta^{j-1}\left(\frac{1}{M_{c}\left(\dot{A} g_{o}, \dot{A} g_{1}, t\right)}-1\right) \\
& \quad=\left(\eta^{\ell}+\eta^{\ell+1}+\cdots+\eta^{j-1}\right)\left(\frac{1}{M_{c}\left(\dot{A} g_{o}, \dot{A} g_{1}, t\right)}-1\right) \\
& \quad=\frac{\eta^{\ell}}{1-\eta}\left(\frac{1}{M_{c}\left(\dot{A} g_{o}, \dot{A} g_{1}, t\right)}-1\right) \longrightarrow 0, \quad \text { as } \ell \longrightarrow \infty .
\end{aligned}
$$
have that

$$
\lim _{\ell, j \rightarrow \infty} M_{c}\left(\dot{A} g_{\ell}, \dot{A} g_{j}, t\right)=1, \quad t \gg 0
$$


Now, again from (34), for $t \gg 0$, we have

$$
\begin{aligned}
& \frac{1}{M_{c}\left(\dot{A} h_{\ell}, \dot{A} h_{\ell+1}, t\right)}-1=\frac{1}{M_{c}\left(\dot{A} \ddot{B}\left(h_{\ell-1}, g_{\ell-1}\right), \dot{A} \ddot{B}\left(h_{\ell}, g_{\ell}\right), t\right)}-1 \\
& \leq \alpha\left(\frac{1}{M_{c}\left(\dot{A} h_{\ell-1}, \dot{A} h_{\ell}, t\right)}-1\right) \\
& +\beta\left[\left(\frac{1}{M_{c}\left(\dot{A} h_{\ell-1}, \dot{A} \ddot{B}\left(h_{\ell-1}, g_{\ell-1}, t\right)\right.}-1\right)+\left(\frac{1}{M_{c}\left(\dot{A} h_{\ell}, \dot{A} \ddot{B}\left(h_{\ell}, g_{\ell}\right), t\right)}-1\right)\right] \\
& +\gamma\left(\frac{1}{M_{c}\left(\dot{A} h_{\ell}, \dot{A} \ddot{B}\left(h_{\ell-1}, g_{\ell-1}\right), t\right) * M_{c}\left(\dot{A} h_{\ell}, \dot{A} \ddot{B}\left(h_{\ell}, g_{\ell}\right), t\right)}-1\right) \\
& =\alpha\left(\frac{1}{M_{c}\left(\dot{A} h_{\ell-1}, \dot{A} h_{\ell}, t\right)}-1\right) \\
& +\beta\left[\left(\frac{1}{M_{c}\left(\dot{A} h_{\ell-1}, \dot{A} h_{\ell}, t\right)}-1\right)+\left(\frac{1}{M_{c}\left(\dot{A} h_{\ell}, \dot{A} h_{\ell+1}, t\right)}-1\right)\right] \\
& +\gamma\left(\frac{1}{M_{c}\left(\dot{A} h_{\ell}, \dot{A} h_{\ell}, t\right) * M_{c}\left(\dot{A} h_{\ell}, \dot{A} h_{\ell+1}, t\right)}-1\right) \\
& =\alpha\left(\frac{1}{M_{c}\left(\dot{A} h_{\ell-1}, \dot{A} h_{\ell}, t\right)}-1\right) \\
& +\beta\left[\left(\frac{1}{M_{c}\left(\dot{A} h_{\ell-1}, \dot{A} h_{\ell}, t\right)}-1\right)+\left(\frac{1}{M_{c}\left(\dot{A} h_{\ell}, \dot{A} h_{\ell+1}, t\right)}-1\right)\right] \\
& +\gamma\left(\frac{1}{M_{c}\left(\dot{A} h_{\ell}, \dot{A} h_{\ell+1}, t\right)}-1\right) \text {. }
\end{aligned}
$$

After simplification, we get that

$$
\begin{array}{r}
\frac{1}{M_{c}\left(\dot{A} h_{\ell}, \dot{A} h_{\ell+1}, t\right)}-1 \leq \eta\left(\frac{1}{M_{c}\left(\dot{A} h_{\ell-1}, \dot{A} h_{\ell}, t\right)}-1\right), \\
\text { for } t \gg 0,
\end{array}
$$

where $\eta$ value is the same as in (37). Similarly, again from (34), for $t \gg 0$, we have

$$
\begin{array}{r}
\frac{1}{M_{c}\left(\dot{A} h_{\ell-1}, \dot{A} h_{\ell}, t\right)}-1 \leq \eta\left(\frac{1}{M_{c}\left(\dot{A} h_{\ell-2}, \dot{A} h_{\ell-1}, t\right)}-1\right) \\
\text { for } t \gg 0,
\end{array}
$$

where $\eta$ value is the same as in (37). Now, from (44) and (45) and by induction, for $t \gg 0$, we have that

$$
\frac{1}{M_{c}\left(\dot{A} h_{\ell}, \dot{A} h_{\ell+1}, t\right)}-1 \leq \eta\left(\frac{1}{M_{c}\left(\dot{A} h_{\ell-1}, \dot{A} h_{\ell}, t\right)}-1\right)
$$

$$
\begin{aligned}
& \leq \eta^{2}\left(\frac{1}{M_{c}\left(\dot{A} h_{\ell-2}, \dot{A} h_{\ell-1}, t\right)}-1\right) \\
& \leq \cdots \leq \eta^{\ell}\left(\frac{1}{M_{c}\left(\dot{A} h_{0}, \dot{A} h_{1}, t\right)}-1\right) \\
& \longrightarrow 0, \quad \text { as } \ell \longrightarrow \infty .
\end{aligned}
$$


Hence, proving that $\left\{\dot{A} h_{\ell}\right\}_{\ell \geq 0}$ is a fuzzy cone contractive $\quad$ Now, for $j>\ell$ and for $t \gg 0$, we have sequence,

$$
\lim _{\ell \longrightarrow \infty} M_{c}\left(\dot{A} h_{\ell}, \dot{A} h_{\ell+1}, t\right)=1, \quad \text { for } t \gg 0 .
$$

$$
\begin{aligned}
& \frac{1}{M_{c}\left(\dot{A} h_{\ell}, \dot{A} h_{j}, t\right)}-1 \\
& \leq\left(\frac{1}{M_{c}\left(\dot{A} h_{\ell}, \dot{A} h_{\ell+1}, t\right)}-1\right)+\left(\frac{1}{M_{c}\left(\dot{A} h_{\ell+1}, \dot{A} h_{\ell+2}, t\right)}-1\right)+\cdots+\left(\frac{1}{M_{c}\left(\dot{A} h_{j-1}, \dot{A} h_{j}, t\right)}-1\right) \\
& \leq \eta^{\ell}\left(\frac{1}{M_{c}\left(\dot{A} h_{0}, \dot{A} h_{1}, t\right)}-1\right)+\eta^{\ell+1}\left(\frac{1}{M_{c}\left(\dot{A} h_{0}, \dot{A} h_{1}, t\right)}-1\right)+\cdots+\eta^{j-1}\left(\frac{1}{M_{c}\left(\dot{A} h_{0}, \dot{A} h_{1}, t\right)}-1\right) \\
& =\left(\eta^{\ell}+\eta^{\ell+1}+\cdots+\eta^{j-1}\right)\left(\frac{1}{M_{c}\left(\dot{A} h_{0}, \dot{A} h_{1}, t\right)}-1\right) \\
& =\frac{\eta^{\ell}}{1-\eta}\left(\frac{1}{M_{c}\left(\dot{A} h_{0}, \dot{A} h_{1}, t\right)}-1\right) \longrightarrow 0, \text { as } \longrightarrow \infty
\end{aligned}
$$

Hence, proving that $\left\{\dot{A} h_{\ell}\right\}$ is a Cauchy sequence,

$$
\lim _{\ell, j \rightarrow \infty} M_{c}\left(\dot{A} h_{\ell}, \dot{A} h_{j}, t\right)=1, \quad \text { for } t \gg 0 .
$$

Since $G$ is complete, $\left\{\dot{A} g_{\ell}\right\}$ and $\left\{\dot{A} h_{\ell}\right\}$ are Cauchy sequences in $G$; therefore $\dot{A} g_{\ell} \longrightarrow g \in G$ and $\dot{A} h_{\ell} \longrightarrow h \in G$ as $\ell \longrightarrow \infty$; that is, $\lim _{\ell \longrightarrow \infty} \dot{A} g_{\ell}=g$ and $\lim _{\ell \longrightarrow \infty} \dot{A} h_{\ell}=h$.
Since $\dot{A}$ is subsequently convergent, $\left\{g_{\ell}\right\}$ has a convergent subsequence. So there exist $g \in G$ and $\left\{g_{\ell(k)}\right\}$ such that $\lim _{k \longrightarrow \infty} g_{\ell(k)}=g$. Since $\dot{A}$ is continuous, $\lim _{k \longrightarrow \infty} g_{\ell(k)}=g$ and $\lim _{k \longrightarrow \infty} \dot{A} g_{\ell(k)}=\dot{A} g$. Now, from (34) and (39), for $t \gg 0$, we have

$$
\begin{aligned}
& \frac{1}{M_{c}(\dot{A} \ddot{B}(g, h), \dot{A} g, t)}-1 \\
& \leq\left(\frac{1}{M_{c}\left(\dot{A} \ddot{B}(g, h), \dot{A} \ddot{B}\left(g_{\ell-1}, h_{\ell-1}\right), t\right)}-1\right)+\left(\frac{1}{M_{c}\left(\dot{A} \ddot{B}\left(g_{\ell-1}, h_{\ell-1}\right), \dot{A} \ddot{B}\left(g_{\ell}, h_{\ell}\right), t\right)}-1\right) \\
& +\left(\frac{1}{M_{c}\left(\dot{A} \ddot{B}\left(g_{\ell}, h_{\ell}\right), \dot{A} g, t\right)}-1\right) \leq \alpha\left(\frac{1}{M_{c}\left(\dot{A} g, \dot{A} g_{\ell-1}, t\right)}-1\right) \\
& +\beta\left[\left(\frac{1}{M_{c}(\dot{A} g, \dot{A} \ddot{B}(g, h), t)}-1\right)+\left(\frac{1}{M_{c}\left(\dot{A} g_{\ell-1}, \dot{A} \ddot{B}\left(g_{\ell-1}, h_{\ell-1}\right), t\right)}-1\right)\right] \\
& +\gamma\left[\frac{1}{M_{c}\left(\ddot{A} g_{\ell-1}, \ddot{A} \ddot{B}(g, h), t\right) * M_{c}\left(\dot{A} g_{\ell-1}, \dot{A} \ddot{B}\left(g_{\ell-1}, h_{\ell-1}\right), t\right)}-1\right] \\
& +\eta^{\ell}\left(\frac{1}{M_{c}\left(\dot{A} g_{0}, \dot{A} g_{1}, t\right)}-1\right)+\left(\frac{1}{M_{c}\left(\dot{A} \ddot{B}\left(g_{\ell}, h_{\ell}\right), \dot{A} g, t\right)}-1\right) .
\end{aligned}
$$


After simplification, for $t \gg 0$, we have that

$$
\begin{aligned}
\frac{1}{M_{c}(\dot{A} \ddot{B}(g, h), \dot{A} g, t)}-1 \leq & \frac{\alpha}{1-\beta}\left(\frac{1}{M_{c}\left(\dot{A} g, \dot{A} g_{\ell-1}, t\right)}-1\right)+\frac{\beta}{1-\beta}\left(\frac{1}{M_{c}\left(\dot{A} g_{\ell-1}, \dot{A} \ddot{B}\left(g_{\ell-1}, h_{\ell-1}\right), t\right)}-1\right) \\
& +\frac{\gamma}{1-\beta}\left(\frac{1}{M_{c}\left(\dot{A} g_{\ell-1}, \dot{A} \ddot{B}(g, h), t\right) * M_{c}\left(\dot{A} g_{\ell-1}, \dot{A} g_{\ell}, t\right)}-1\right) \\
& +\frac{1}{1-\beta} \eta^{\ell}\left(\frac{1}{M_{c}\left(\dot{A} g_{0}, \dot{A} g_{1}, t\right)}-1\right)+\frac{1}{1-\beta}\left(\frac{1}{M_{c}\left(\dot{A} \ddot{B}\left(g_{\ell}, h_{\ell}\right), \dot{A} g, t\right)}-1\right) \longrightarrow 0, \quad \text { as } \ell \longrightarrow \infty .
\end{aligned}
$$

Hence, we get that $M_{c}(\dot{A} \ddot{B}(g, h), \dot{A} g, t)=1$, and this implies that $\dot{A} \ddot{B}(g, h)=\dot{A} g$. Since $\dot{A}$ is one-one, $\ddot{B}(g, h)=g$.

Now, again from (34) and (46), for $t \gg 0$, we have

$$
\begin{aligned}
& \frac{1}{M_{c}(\dot{A} \ddot{B}(h, g), \dot{A} h, t)}-1 \\
& \leq\left(\frac{1}{M_{c}\left(\dot{A} \ddot{B}(h, g), \dot{A} \ddot{B}\left(h_{\ell-1}, g_{\ell-1}\right), t\right)}-1\right)+\left(\frac{1}{M_{c}\left(\dot{A} \ddot{B}\left(h_{\ell-1}, g_{\ell-1}\right), \dot{A} \ddot{B}\left(h_{\ell}, g_{\ell}\right), t\right)}-1\right) \\
& \quad+\left(\frac{1}{M_{c}\left(\dot{A} \ddot{B}\left(h_{\ell}, g_{\ell}\right), \dot{A} h, t\right)}-1\right) \leq \alpha\left(\frac{1}{M_{c}\left(\dot{A} h, \dot{A} h_{\ell-1}, t\right)}-1\right) \\
& +\beta\left[\left(\frac{1}{M_{c}(\dot{A} h, \dot{A} \ddot{B}(h, g), t)}-1\right)+\left(\frac{1}{M_{c}\left(\dot{A} h_{\ell-1}, \dot{A} \ddot{B}\left(h_{\ell-1}, g_{\ell-1}\right), t\right)}-1\right)\right] \\
& \quad+\gamma\left(\frac{1}{M_{c}\left(\dot{A} h_{\ell-1}, \dot{A} \ddot{B}(h, g), t\right) * M_{c}\left(\dot{A} h_{\ell-1}, \dot{A} \ddot{B}\left(h_{\ell-1}, g_{\ell-1}\right), t\right)}-1\right) \\
& \quad+\eta^{\ell}\left(\frac{1}{M_{c}\left(\dot{A} h_{0}, \dot{A} h_{1}, t\right)}-1\right)+\left(\frac{1}{M_{c}\left(\dot{A} \ddot{B}\left(h_{\ell}, g_{\ell}\right), \dot{A} h, t\right)}-1\right) .
\end{aligned}
$$

After simplification, for $t \gg 0$, we have that

$$
\begin{aligned}
\frac{1}{M_{c}(\dot{A} \ddot{B}(h, g), \dot{A} h, t)}-1 \leq & \frac{\alpha}{1-\beta}\left(\frac{1}{M_{c}\left(\dot{A} h, \dot{A} h_{\ell-1}, t\right)}-1\right)+\frac{\beta}{1-\beta}\left(\frac{1}{M_{c}\left(\dot{A} h_{\ell-1}, \dot{A} \ddot{B}\left(h_{\ell-1}, g_{\ell-1}\right), t\right)}-1\right) \\
& +\frac{\gamma}{1-\beta}\left(\frac{1}{M_{c}\left(\dot{A} h_{\ell-1}, \dot{A} \ddot{B}(h, g), t\right) * M_{c}\left(\dot{A} h_{\ell-1}, \ddot{A} h_{\ell}, t\right)}-1\right) \\
& +\frac{1}{1-\beta} \eta^{\ell}\left(\frac{1}{M_{c}\left(\dot{A} h_{0}, \dot{A} h_{1}, t\right)}-1\right)+\frac{1}{1-\beta}\left(\frac{1}{M_{c}\left(\dot{A} \ddot{B}\left(h_{\ell}, g_{\ell}\right), \dot{A} h, t\right)}-1\right) \longrightarrow 0, \quad \text { as } \ell \longrightarrow \infty
\end{aligned}
$$


Hence, we get that $M_{c}(\dot{A} \ddot{B}(h, g), \dot{A} h, t)=1$ for $t \gg 0$, and this implies that $\dot{A} \ddot{B}(h, g)=\dot{A} h$. Since $\dot{A}$ is one-one, $\ddot{B}(h, g)=h$.
For uniqueness, let $\left(g_{1}, h_{1}\right)$ and $\left(h_{1}, g_{1}\right)$ be coupled fixed-point pairs in $G \times G$ such that $\ddot{B}\left(g_{1}, h_{1}\right)=g_{1}$ and $\ddot{B}\left(h_{1}, g_{1}\right)=h_{1}$. Now, from (34), for $t \gg 0$, we have

$$
\begin{aligned}
\frac{1}{M_{c}\left(\dot{A} g, \dot{A} g_{1}, t\right)}-1= & \left(\frac{1}{M_{c}\left(\dot{A} \ddot{B}(g, h), \dot{A} \ddot{B}\left(g_{1}, h_{1}\right), t\right)}-1\right) \\
\leq & \alpha\left(\frac{1}{M_{c}\left(\dot{A} g, \dot{A} g_{1}, t\right)}-1\right)+\beta\left[\left(\frac{1}{M_{c}(\dot{A} g, \dot{A} \ddot{B}(g, h), t)}-1\right)+\left(\frac{1}{M_{c}\left(\dot{A} g_{1}, \dot{A} \ddot{B}\left(g_{1}, h_{1}\right), t\right)}-1\right)\right] \\
& +\gamma\left(\frac{1}{M_{c}\left(\dot{A} g_{1}, \dot{A} \ddot{B}(g, h), t\right) * M_{c}\left(\dot{A} g_{1}, \dot{A} \ddot{B}\left(g_{1}, h_{1}\right), t\right)}-1\right) \\
= & (\alpha+\gamma)\left(\frac{1}{M_{c}\left(\dot{A} g, \dot{A} g_{1}, t\right)}-1\right) \\
= & (\alpha+\gamma)\left(\frac{1}{M_{c}\left(\dot{A} \ddot{B}(g, h), \dot{A} \ddot{B}\left(g_{1}, h_{1}\right), t\right)}-1\right) \\
\leq & (\alpha+\gamma)^{2}\left(\frac{1}{M_{c}\left(\dot{A} g, \dot{A} g_{1}, t\right)}-1\right) \\
\leq & \cdots \leq(\alpha+\gamma)^{\ell}\left(\frac{1}{M_{c}\left(\dot{A} g, \dot{A} g_{1}, t\right)}-1\right) \longrightarrow 0, \quad \text { as } \ell \longrightarrow \infty .
\end{aligned}
$$

Hence, we get that $M_{c}\left(\dot{A} g, \dot{A} g_{1}, t\right)=1$, and this implies that $g=g_{1}$. Similarly, again from (34), for $t \gg 0$, we have

$$
\begin{aligned}
\frac{1}{M_{c}\left(\dot{A} h, \dot{A} h_{1}, t\right)}-1= & \left(\frac{1}{M_{c}\left(\dot{A} \dot{B}(h, g), \dot{A} \ddot{B}\left(h_{1}, g_{1}\right), t\right)}-1\right) \\
\leq & \alpha\left(\frac{1}{M_{c}\left(\dot{A} h, \dot{A} h_{1}, t\right)}-1\right)+\beta\left[\left(\frac{1}{M_{c}(\dot{A} h, \dot{A} \ddot{B}(h, g), t)}-1\right)+\left(\frac{1}{M_{c}\left(\dot{A} h_{1}, \dot{A} \ddot{B}\left(h_{1}, g_{1}\right), t\right)}-1\right)\right] \\
& +\gamma\left(\frac{1}{M_{c}\left(\dot{A} h_{1}, \dot{A} \ddot{B}(h, g), t\right) * M_{c}\left(\dot{A} h_{1}, \dot{A} \ddot{B}\left(h_{1}, g_{1}\right), t\right)}-1\right) \\
= & (\alpha+\gamma)\left(\frac{1}{M_{c}\left(\dot{A} h, \dot{A} h_{1}, t\right)}-1\right) \\
= & (\alpha+\gamma)\left(\frac{1}{M_{c}\left(\dot{A} \ddot{B}(h, g), \dot{A} \ddot{B}\left(h_{1}, g_{1}\right), t\right)}-1\right) \\
\leq & (\alpha+\gamma)^{2}\left(\frac{1}{M_{c}\left(\dot{A} h, \dot{A} h_{1}, t\right)}-1\right) \\
\leq & \cdots \leq(\alpha+\gamma)^{\ell}\left(\frac{1}{M_{c}\left(\dot{A} h, \dot{A} h_{1}, t\right)}-1\right) \longrightarrow 0
\end{aligned}
$$


Hence, we get that $M_{c}\left(\dot{A} h, \dot{A} h_{1}, t\right)=1$ for $t \gg 0$, and this implies that $h=h_{1}$.

Corollary 4. Let $\ddot{B}: G \times G \longrightarrow G$ be a mapping in a complete FCM-space $\left(G, M_{c}, *\right)$ in which $M_{c}$ is triangular and $A$ is a continuous, one-one, and subsequently convergent selfmapping on $G$, that is, $\dot{A}: G \longrightarrow G$, satisfying

$$
\begin{aligned}
& \frac{1}{M_{c}(\dot{A} \ddot{B}(g, h), \dot{A} \ddot{B}(\xi, \eta), t)}-1 \leq \alpha\left(\frac{1}{M_{c}(\dot{A} g, \dot{A} \xi, t)}-1\right) \\
& \quad+\gamma\left[\left(\frac{1}{M_{c}(\dot{A} \xi, \dot{A} \ddot{B}(g, h), t) * M_{c}(\dot{A} \xi, \dot{A} \ddot{B}(\xi, \eta), t)}-1\right)\right],
\end{aligned}
$$

for all $g, h, \xi, \eta \in G, t \gg 0$, and $\alpha, \gamma \in[0,1]$ with $\alpha+\gamma<1$. Then $\ddot{B}$ has a unique coupled FP. Also, if $\dot{A}$ converges sequently, then, for each $g_{0} \in G$, the iterative sequence $\left\{\ddot{B}^{\ell} g_{o}\right\}$ converges to this coupled FP.

If we use $\dot{A}$ as an identity self-mapping, that is, $\dot{A}=I$ in Theorem 2, then we get the following corollary.

Corollary 5. Let $\ddot{B}: G \times G \longrightarrow G$ be a mapping in a complete FCM-space $\left(G, M_{c}, *\right)$ in which $M_{c}$ is triangular, satisfying

$$
\begin{aligned}
\frac{1}{M_{c}(\ddot{B}(g, h), \ddot{B}(\xi, \eta), t)}-1 \leq & \alpha\left(\frac{1}{M_{c}(g, \xi, t)}-1\right) \\
& +\beta\left[\left(\frac{1}{M_{c}(g, \ddot{B}(g, h), t)}-1\right)+\left(\frac{1}{M_{c}(\xi, \ddot{B}(\xi, \eta), t)}-1\right)\right] \\
& +\gamma\left[\left(\frac{1}{M_{c}(\xi, \ddot{B}(g, h), t) * M_{c}(\xi, \ddot{B}(\xi, \eta), t)}-1\right)\right],
\end{aligned}
$$

for all $g, h, \xi, \eta \in G, \quad t \gg 0$, and $\alpha, \beta, \gamma \in[0,1]$ with $\alpha+2 \beta+\gamma<1$. Then $\ddot{B}$ has a unique coupled FP in $G$.

Example 2. Let $G=\{0\} \cup\{(1 / 2),(1 / 3),(1 / 4), \ldots\}$, and let $M_{c}: G^{2} \times(0, \infty) \longrightarrow[0,1]$ be defined as

$$
M_{c}(g, h, t)=\frac{t}{t+d(g, h)}, \quad \text { where } d(g, h)=|g-h| \text {, }
$$

for all $g, h \in G$ and $t>0$. Then, it could be verified that $M_{c}$ is triangular and $\left(G, M_{c}, *\right)$ is a complete FCM-space. We define $\ddot{B}: G \times G \longrightarrow G$ by $\ddot{B}(0,0)=0$ and $\ddot{B}((1 / n),(1 / \ell))=$ $1 /(n+\ell+1)$ for all $n, \ell \geq 2$; and a mapping $\dot{A}: G \longrightarrow G$ is defined as $\dot{A}(0)=0$ and $\ddot{A}(1 / n)=\left(1 / n^{n}\right)$. Then, by using (58), for $t \gg 0$, we have

$$
\begin{aligned}
& \frac{1}{M_{c}(\dot{A} \ddot{B}((1 / n),(1 / l)), \dot{A} \ddot{B}((1 / p),(1 / q)), t)}-1 \\
& =\frac{1}{t}\left[d\left(\dot{A} \ddot{B}\left(\frac{1}{n}, \frac{1}{\ell}\right), \dot{A} \ddot{B}\left(\frac{1}{p}, \frac{1}{q}\right)\right)\right] \\
& =\frac{1}{t}\left[\frac{1}{(n+\ell+1)^{n+\ell+1}}-\frac{1}{(p+q+1)^{p+q+1}}\right] \text {. }
\end{aligned}
$$

Let $(1 / t)\left(1 /(n+\ell+1)^{n+\ell+1}\right) \leq(1 / 5 t)\left(\left(2 / n^{n}\right)-(1 /(n+\ell\right.$ $\left.\left.+1)^{n+\ell+1}\right)\right)$. Then, from (??), for $t \gg 0$, we have

$$
\begin{aligned}
\frac{1}{t} & {\left[\frac{1}{(n+\ell+1)^{n+\ell+1}}-\frac{1}{(p+q+1)^{p+q+1}}\right] } \\
\leq & \frac{1}{5 t}\left[\left(\frac{2}{n^{n}}-\frac{1}{(n+\ell+1)^{n+\ell+1}}\right)-\left(\frac{2}{p^{p}}-\frac{1}{(p+q+1)^{p+q+1}}\right)\right] \\
= & \frac{1}{t}\left[\frac{1}{5 n^{n}}+\frac{1}{5 n^{n}}-\frac{1}{10(n+\ell+1)^{n+\ell+1}}-\frac{1}{10(n+\ell+1)^{n+\ell+1}}\right] \\
& -\frac{1}{t}\left[\frac{1}{5 p^{p}}+\frac{1}{5 p^{p}}-\frac{1}{10(p+q+1)^{p+q+1}}-\frac{1}{10(p+q+1)^{p+q+1}}\right] \\
= & \frac{1}{t}\left(\frac{1}{5 n^{n}}-\frac{1}{5 p^{p}}\right)+\frac{1}{t}\left[\frac{1}{5 n^{n}}-\frac{1}{10(n+\ell+1)^{n+\ell+1}}-\frac{1}{10(n+\ell+1)^{n+\ell+1}}\right] \\
& -\frac{1}{t}\left[\frac{1}{5 p^{p}}-\frac{1}{10(p+q+1)^{p+q+1}}-\frac{1}{10(p+q+1)^{p+q+1}}\right] \\
= & \frac{1}{t}\left(\frac{1}{5 n^{n}}-\frac{1}{5 p^{p}}\right)+\frac{1}{t}\left[\left(\frac{1}{10 n^{n}}-\frac{1}{10(n+\ell+1)^{n+\ell+1}}\right)+\left(\frac{1}{10 p^{p}}-\frac{1}{10(p+q+1)^{p+q+1}}\right)\right] \\
& +\frac{1}{t}\left[\frac{1}{10 n^{n}}-\frac{3}{10 p^{p}}+\frac{1}{10(p+q+1)^{p+q+1}}-\frac{1}{10(n+\ell+1)^{n+\ell+1}}\right] .
\end{aligned}
$$


We have

Therefore,

$$
\begin{aligned}
& \frac{1}{t}\left[\frac{1}{10 n^{n}}-\frac{3}{10 p^{p}}+\frac{3}{10(p+q+1)^{p+q+1}}-\frac{1}{10(n+\ell+1)^{n+\ell+1}}\right] \\
& \quad \leq \frac{1}{t}\left[\frac{1}{\left(10 p^{p}-10(n+\ell+1)^{n+\ell+1}\right) *\left(10 p^{p}-10(p+q+1)^{p+q+1}\right)}\right] .
\end{aligned}
$$

$$
\begin{aligned}
\frac{1}{M_{c}(\dot{A} \ddot{B}((1 / n),(1 / \ell)), \dot{A} \ddot{B}((1 / p),(1 / q)), t)}= & \frac{1}{t}\left(\frac{1}{(n+\ell+1)^{n+\ell+1}}-\frac{1}{(p+q+1)^{p+q+1}}\right) \\
\leq & \frac{1}{t}\left(\frac{1}{5 n^{n}}-\frac{1}{5 p^{p}}\right) \\
& +\frac{1}{t}\left[\left(\frac{1}{10 n^{n}}-\frac{1}{10(n+\ell+1)^{n+\ell+1}}\right)+\left(\frac{1}{10 p^{p}}-\frac{1}{10(p+q+1)^{p+q+1}}\right)\right] \\
& +\frac{1}{t}\left[\frac{1}{\left(10 p^{p}-10(n+\ell+1)^{n+\ell+1}\right) *\left(10 p^{p}-10(p+q+1)^{p+q+1}\right)}\right] \\
= & \frac{1}{5}\left(\frac{1}{M_{c}(\dot{A}(1 / n), \dot{A}(1 / p), t)}-1\right) \\
& +\frac{1}{10}\left[\left(\frac{1}{M_{c}(\dot{A}(1 / n), \dot{A} \ddot{B}((1 / n),(1 / \ell)), t)}-1\right)+\left(\frac{1}{M_{c}(\dot{A}(1 / p), \dot{A} \ddot{B}((1 / p),(1 / q)), t)}-1\right)\right] \\
& +\frac{1}{10}\left[\frac{1}{M_{c}(\dot{A}(1 / p), \dot{A} \ddot{B}((1 / n),(1 / \ell)), t) * M_{c}(\dot{A}(1 / p), \dot{A} \ddot{B}((1 / p),(1 / q)), t)}-1\right] .
\end{aligned}
$$

This implies that

$$
\begin{aligned}
\frac{1}{M_{c}(\dot{A} \ddot{B}((1 / n),(1 / \ell)), \dot{A} \ddot{B}((1 / p),(1 / q)), t)}-1 \leq & \frac{1}{5}\left(\frac{1}{M_{c}(\dot{A}(1 / n), \dot{A}(1 / p), t)}-1\right) \\
& +\frac{1}{10}\left[\left(\frac{1}{M_{c}(\dot{A}(1 / n), \dot{A} \ddot{B}((1 / n),(1 / \ell)), t)}-1\right)+\left(\frac{1}{M_{c}(\dot{A}(1 / p), \dot{A} \ddot{B}((1 / p),(1 / q)), t)}-1\right)\right] \\
& +\frac{1}{10}\left[\frac{1}{M_{c}(\dot{A}(1 / p), \dot{A} \ddot{B}((1 / n),(1 / \ell)), t) * M_{c}(\dot{A}(1 / p), \dot{A} \ddot{B}((1 / p),(1 / q)), t)}-1\right] .
\end{aligned}
$$

Thus, inequality (63) satisfies all the conditions of Theorem 2 with $\alpha=(1 / 5)$ and $\beta=\gamma=(1 / 10)$ and $\ddot{B}$ has a unique coupled FP; that is, $\ddot{B}(0,0)=0$ and $\dot{A}(0)=0$.

\section{Application}

In this section, we present an application on Volterra integral equations (VIEs) to support our main work. We prove that the solution of the two Volterra integral equations has a common FP of the integral operators of $\ddot{B}$ and $\dot{A}$ which are defined in (71) to support our result, that is, Theorem 1 . Now we shall define the following terms by using supremum norm to justify our work. Let $G=C([0,1], R)$ be the space of all continuous real-valued on $[0,1]$. The supremum norm on $\mathrm{G}$ is defined as

$$
\|g\|=\sup _{r \in[0,1]}\|g(r)\|, \quad \text { where } g \in C([0,1], R)
$$

Now, we define a metric $d: G \times G \longrightarrow R$ by 


$$
\begin{array}{r}
d(g, h)=\sup _{r \in[0,1]}|g(r)-h(r)|=\|g(r)-h(r)\|, \\
\text { where } g, h \in C([0,1], R) .
\end{array}
$$

As $*$ is continuous $t$-norm, for all $\alpha, \beta \in[0,1]$, we have $\alpha * \beta=\alpha \beta$, and a fuzzy metric $M_{c}: G \times G \times(0, \infty) \longrightarrow[0,1]$ is defined as

$$
M(g, h, t)=\frac{t}{t+d(g, h)}, \quad \text { where } d(g, h)=\|g-h\|,
$$

for $t>0$ and $g, h \in C([0,1], R)$. Then, one can easily prove that $M_{c}$ is triangular and $\left(G, M_{c}, *\right)$ is a complete fuzzy cone metric space.

Now we present the two VIEs for a common solution to uphold our result.

Theorem 3. The two VIEs are

$$
\begin{aligned}
& g(\zeta)=\hbar_{1}(\zeta)+\int_{0}^{1} K_{1}(\zeta, s, g(s)) \mathrm{d} s \\
& h(\zeta)=\hbar_{2}(\zeta)+\int_{0}^{1} K_{2}(\zeta, s, h(s)) \mathrm{d} s
\end{aligned}
$$

where $\zeta \in[0,1] \subset R$ and $\hbar_{1}, \hbar_{2} \in G$. Assume that $K_{1}, K_{2}:[0,1]^{2} \times R \longrightarrow R$ such that $U_{(g, h)}, V_{(\xi, \eta)} \in G$ for $g, \eta \in U$, and $h, \xi \in V$ and $U, V \subset G$. Therefore, we define

$$
\begin{aligned}
& U_{(g, h)}(\zeta)=\int_{0}^{1} K_{1}(\zeta, s,(g, h)(s)) \mathrm{d} s \\
& V_{(\xi, \eta)}(\zeta)=\int_{0}^{1} K_{2}(\zeta, s,(\xi, \eta)(s)) \mathrm{d} s, \quad \forall \zeta \in[0,1] .
\end{aligned}
$$

If there exist $\lambda \in[0,1]$ such that

$$
\left\|\left(U_{(g, h)}^{*}+\hbar_{1}\right)-\left(V_{(\xi, \eta)}^{*}+\hbar_{2}\right)\right\| \leq \lambda N\left(U_{(g, h)}, V_{(\xi, \eta)}\right),
$$

where

$$
N\left(U_{(g, h)}, V_{(\xi, \eta)}\right)=\max \left\{\begin{array}{c}
\left\|U_{g}-V_{\xi}\right\| \\
\left(\left\|U_{(g, h)}^{*}+U_{\hbar_{1}}-U_{g}\right\|+\left\|V_{(\xi, \eta)}^{*}+V_{\hbar_{2}}-V_{\xi}\right\|\right) \\
\left(\left\|V_{(\xi, \eta)}^{*}+V_{\hbar_{2}}-U_{g}\right\|+\left\|U_{(g, h)}^{*}+U_{\hbar_{1}}-V_{\xi}\right\|\right)
\end{array}\right\}
$$

where $U_{(g, h)}^{*}, V_{(\xi, \eta)}^{*}, U_{\hbar_{1}}, V_{\hbar_{2}}, U_{g}, V_{\xi} \in G$, then the two VIEs (67) have a unique common solution in $G$.

Proof. Define operators $\ddot{B}: G \times G \longrightarrow G$ and $\dot{A}: G \longrightarrow G$ :

$$
\begin{aligned}
\ddot{B}(g, h) & =U_{(g, h)}+\hbar_{1}, \\
\dot{A} g & =U_{g}, \\
\ddot{B}(\xi, \eta) & =V_{(\xi, \eta)}+\hbar_{2}, \\
\dot{A} \xi & =V_{\xi} .
\end{aligned}
$$

$$
\begin{aligned}
& \dot{A} \ddot{B}(g, h)=\dot{A}\left(U_{(g, h)}+\hbar_{1}\right)=U_{U_{(g, h)}}+U_{\hbar_{1}}=U_{(g, h)}^{*}+U_{\hbar_{1}}, \\
& \dot{A} \ddot{B}(\xi, \eta)=\dot{A}\left(V_{(\xi, \eta)}+\hbar_{2}\right)=V_{V_{(\xi, \eta)}}+V_{\hbar_{2}}=V_{(\xi, \eta)}^{*}+V_{\hbar_{2}},
\end{aligned}
$$

where $U_{U_{(g, h)}}=U_{(g, h)}^{*}$ and $V_{V_{(\xi, \eta)}}=V_{(\xi, \eta)}^{*}$. Now, we may have the three following cases:

(1) If $N\left(U_{(g, h)}, V_{(\xi, \eta)}\right)=\left\|U_{g}-V\right\|_{\xi}$, then, from (66), (71) and (72), we have

Then,

$$
\begin{aligned}
\frac{1}{M_{c}(\dot{A} \ddot{B}(g, h), \dot{A} \ddot{B}(\xi, \eta), t)}-1 & =\frac{1}{t}\|\dot{A} \ddot{B}(g, h)-\dot{A} B(\xi, \eta)\| \\
& \leq \frac{\lambda}{t} N\left(U_{(g, h)}, V_{(\xi, \eta)}\right) \\
& =\frac{\lambda}{t}\left\|U_{g}-V_{\xi}\right\| \\
& =\lambda\left(\frac{1}{M_{c}(\dot{A} g, \dot{A} \xi, t)}-1\right), \quad \text { for } t \gg 0,
\end{aligned}
$$


for $g, \eta \in U$, and $h, \xi \in V$. Hence, operators $\dot{A}$ and $\ddot{B}$ satisfy all the conditions of Theorem 1 with $\lambda=\alpha$ and $\beta=\gamma=0$ in (5). Thus, the two VIEs in (67) have a unique common solution in $G$. Then integral equations have a unique common solution.
(2) If $\quad N\left(U_{(g, h)}, V_{(\xi, \eta)}\right)=\left(\left\|U_{(g, h)}^{*}+U_{\hbar_{1}}-U_{g}\right\|+\right.$ $\left.\left\|V_{(\xi, \eta)}^{*}+V_{\hbar_{2}}-V_{\xi}\right\|\right)$, then, from (66), (71) and (72), we have

$$
\begin{aligned}
\frac{1}{M_{c}(\dot{A} \ddot{B}(g, h), \dot{A} \ddot{B}(\xi, \eta), t)}-1 & =\frac{1}{t}\|\dot{A} \ddot{B}(g, h)-\dot{A} \ddot{B}(\xi, \eta)\| \\
& \leq \frac{\lambda}{t} N\left(U_{(g, h)}, V_{(\xi, \eta)}\right) \\
& =\frac{\lambda}{t}\left(\left\|U_{(g, h)}^{*}+U_{\hbar_{1}}-U_{g}\right\|+\left\|V_{(\xi, \eta)}^{*}+V_{\hbar_{2}}-V_{\xi}\right\|\right) \\
& =\lambda\left[\left(\frac{1}{M_{c}(\dot{A} g, \dot{A} \ddot{B}(g, h), t)}-1\right)+\left(\frac{1}{M_{c}(\dot{A} \xi, \dot{A} \ddot{B}(\xi, \eta), t)}-1\right)\right], \text { for } t \gg 0,
\end{aligned}
$$

for $g, \eta \in U$, and $h, \xi \in V$. Hence, operators $\dot{A}$ and $\ddot{B}$ satisfy all the conditions of Theorem 1 with $\lambda=\beta$ and $\alpha=\gamma=0$ in (5). Thus, the two VIEs in (67) have a unique common solution in G. Then integral equations have a unique common solution.
(3) If $\quad N\left(U_{(g, h)}, V_{(\xi, \eta)}\right)=\left(\left\|V_{(\xi, \eta)}^{*}+V_{\hbar_{2}}-A_{g}\right\|+\right.$ $\left.\left\|U_{(g, h)}^{*}+U_{\hbar_{1}}-V_{\xi}\right\|\right)$, then, from (66), (71) and (72), we have

$$
\begin{aligned}
\frac{1}{M_{c}(\dot{A} \ddot{B}(g, h), \dot{A} \ddot{B}(\xi, \eta), t)}-1 & =\frac{1}{t}\|\dot{A} \ddot{B}(g, h)-\dot{A} \ddot{B}(\xi, \eta)\| \\
& \leq \frac{\lambda}{t} N\left(U_{(g, h)}, V_{(\xi, \eta)}\right) \\
& =\frac{\lambda}{t}\left(\left\|V_{(\xi, \eta)}^{*}+V_{\hbar_{2}}-U_{g}\right\|+\left\|U_{(g, h)}^{*}+U_{\hbar_{1}}-V_{\xi}\right\|\right) \\
& =\lambda\left[\left(\frac{1}{M_{c}(\dot{A} g, \dot{A} \ddot{B}(\xi, \eta), t)}-1\right)+\left(\frac{1}{M_{c}(\dot{A} \xi, \dot{A} \ddot{B}(g, h), t)}-1\right)\right], \quad \text { for } t \gg 0,
\end{aligned}
$$

for $g, \eta \in U$, and $h, \xi \in V$. Hence, operators $\dot{A}$ and $\ddot{B}$ satisfy all the conditions of Theorem 1 with $\lambda=\gamma$ and $\alpha=\beta=0$ in (5). Thus, the two VIEs in (67) have a unique common solution in $G$. Then integral equations have a unique common solution.

\section{Conclusion}

We presented the concept of coupled FP results depending on another function in FCM-spaces and prove some unique coupled FP-theorems by using "the triangular property of fuzzy cone metric" by using different contractive type conditions. The other function is a self-mapping that is continuous, one-one, and subsequently convergent in FCMspaces. Further, we presented two Volterra integral equations to uplift our main work. By using this new concept, one can prove more different contractive type coupled FP results depending on another function in complete FCM-spaces. Instead of Volterra integral equations, researchers can use different types of applications such as Lebesgue integral equations, Riemann integral equations, and nonlinear integral equations to support their findings.

\section{Data Availability}

Data sharing is not applicable to this article as no data sets were generated or analysed during the current study.

\section{Conflicts of Interest}

The authors declare that there are no conflicts of interest regarding the publication of this paper. 


\section{Acknowledgments}

The authors are grateful to the Deanship of Scientific Research, King Saud University, for funding through Vice Deanship of Scientific Research Chairs.

\section{References}

[1] S. Banach, "Surles operation dans les ensembles abstraits et leur application aux equations integral," Fundamenta Mathematicae, vol. 3, no. 1, pp. 133-181, 1922.

[2] R. Kannan, "Some results on fixed points," Bulletin of the Calcutta Mathematical Society, vol. 60, pp. 71-76, 1968.

[3] S. K. Chatterjea, "Fixed point theorems," Comptes rendus de l'Academie bulgare des Sciences, vol. 25, pp. 727-730, 1972.

[4] S. Reich, "Remarks concerning contraction mappings," Canadian Mathematical Bulletin, vol. 14, pp. 121-124, 1971.

[5] S. Reich, "Remarks on fixed points," Atti della Accademia Nazionale dei Lincei. Classe di Scienze Fisiche, Matematiche e Naturali Rendiconti, vol. 52, pp. 689-697, 1972.

[6] H. Covitz and S. B. Nadler, "Multi-valued contraction mappings in generalized metric spaces," Israel Journal of Mathematics, vol. 8, pp. 5-11, 1970.

[7] P. Z. Daffer and H. Kaneko, "Fixed points of generalized contractive multi-valued mappings," Journal of Mathematical Analysis and Applications, vol. 192, no. 2, pp. 655-666, 1995.

[8] A. Kaewkhao and K. Neammanee, "Fixed point theorems of multivalued Zamfirescu mapping," Journal of Mathematics Research, vol. 2, no. 2, pp. 150-156, 2010.

[9] L. Huang and X. Zhang, "Cone metric spaces and fixed point theorems of contractive mappings," Journal of Mathematical Analysis and Applications, vol. 332, no. 2, pp. 1468-1476, 2007.

[10] S. Rezapour and R. Hamlbarani, "Some note on the paper cone metric spaces and fixed point theorems of contractive mappings," Journal of Mathematical Analysis and Applications, vol. 345, no. 2, pp. 719-724, 2008.

[11] S. H. Alnafei, S. Radenovi c, and N. Shahzad, "Fixed point theorems for mappings with convex diminishing diameters on cone metric spaces," Applied Mathematics Letters, vol. 24, no. 12, pp. 2162-2166, 2011.

[12] Z. Kadelburg and S. Radenovic, "A note on various types of cones and fixed point results in cone metric spaces," Asian Journal of Mathematics and Applications, vol. 2013, 2013.

[13] A. Kumar, S. Rathee, and N. Kumar, "The point of coincidence and common fixed point for three mappings in cone metric paces," Journal of Applied Mathematics, vol. 2013, Article ID 146794, 2013.

[14] S. Radenovic, "A pair of nonself mappings in cone metric space," Kragujevac Journal of Mathematics, vol. 36, no. 2, pp. 189-198, 2012.

[15] L. A. Zadeh, "Fuzzy sets," Information and Control, vol. 8, no. 3, pp. 338-353, 1965.

[16] V. Ivanova and B. Zlatanov, "Implementation of fuzzy functions aimed at fairer grading of students' tests," Education Sciences, vol. 9, no. 3, 2019.

[17] V. Ivanova and B. Zlatanov, "Application of fuzzy logic in online test evaluation in English as a foreign language at university level," AIP Conference Proceedings, vol. 2172, no. 1, 2019.

[18] N. Rusmiari, D. Putra, and A. Sasmita, "Fuzzy logic method for evaluation of diffculty level of exam and student graduation," International Journal of Computer Science, vol. 10, no. 2, pp. 223-229, 2013.
[19] A. Sobrino, "Fuzzy logic and education: teaching the basics of fuzzy logic through an example (by way of cycling)," Education Sciences, vol. 3, pp. 75-97, 2013.

[20] O. Kramosil and J. Michalek, "Fuzzy metric and statistical metric spaces," Kybernetika, vol. 11, no. 5, pp. 336-344, 1975.

[21] A. George and P. Veeramani, "On some results in fuzzy metric spaces,” Fuzzy Sets and Systems, vol. 64, no. 3, pp. 395-399, 1994.

[22] V. Gregori and A. Sapena, "On fixed point theorems in fuzzy metric spaces," Fuzzy Sets and Systems, vol. 125, pp. 245-252, 2002.

[23] X. Li, S. U. Rehman, S. U. Khan, H. Aydi, N. Hussain, and J. Ahmad, "Strong coupled fixed point analysis in fuzzy metric spaces with application to Urysohn integral equations," $D y$ namic Systems and Applications, vol. 30, no. 2, pp. 197-218, 2002.

[24] S. U. Rehman, R. Chinram, and C. Boonpok, "Rational type fuzzy-contraction results in fuzzy metric spaces with an application," Journal of Mathematics, vol. 2021, Article ID 6644491, 15 pages, 2021.

[25] T. Öner, M. B. Kandemir, and B. Tanay, "Fuzzy cone metric spaces," Journal of Nonlinear Sciences and Applications, vol. 8, no. 5, pp. 610-616, 2015.

[26] S. Ur Rehman and H.-X. Li, "Fixed point theorems in fuzzy cone metric spaces," The Journal of Nonlinear Sciences and Applications, vol. 10, no. 11, pp. 5763-5769, 2017.

[27] S. Jabeen, S. U. Rehman, Z. Zheng, and W. Wei, "Weakly compatible and Quasi-contraction results in fuzzy cone metric spaces with application to the Urysohn type integral equations," Advances in Differential Equations, vol. 2020, p. $16,2020$.

[28] G. X. Chen, S. Jabeen, S. U. Rehman et al., "Coupled fixed point analysis in fuzzy cone metric spaces with application to nonlinear integral equations," Advances in Differential Equations, vol. 2020, p. 25, 2020.

[29] S. U. Rehman and H. Aydi, "Rational fuzzy cone contractions on fuzzy cone metric spaces with an application to Fredholm integral equations," Journal of Function Spaces, vol. 2021, Article ID 5527864, 13 pages, 2021.

[30] D. Guo and V. Lakshmikantham, "Coupled fixed points of nonlinear operators with applications," Nonlinear Analysis: Theory, Methods and Applications, vol. 11, no. 5, pp. 623-632, 1987.

[31] T. G. Bhaskar and V. Lakshmikantham, "Fixed point theorems in partially ordered metric spaces and applications," Nonlinear Analysis: Theory, Methods and Applications, vol. 65, no. 7, pp. 1379-1393, 2006.

[32] V. Lakshmikantham and L. Ciric, "Coupled fixed point theorems for nonlinear contractions in partially ordered metric spaces," Nonlinear Analysis: Theory, Methods and Applications, vol. 70, no. 12, pp. 4341-4349, 2009.

[33] S. Sedghi, I. Altun, and N. Shobe, "Coupled fixed point theorems for contractions in fuzzy metric spaces," Nonlinear Analysis: Theory, Methods and Applications, vol. 72, no. 3-4, pp. 1298-1304, 2010.

[34] S. Moradi, "Kannan fixed point theorem on complete metric spaces and generalized metric spaces depended on another function," arXiv: 0903. 1577v1 [math.FA], 2009.

[35] B. Schweizer and A. Sklar, "Probabilistic metric spaces," NorthHolland Series in Probability and Applied Mathematics, NorthHolland, New York, NY, USA, 1983. 